\title{
A Three-Dimensional Scale-Adaptive Turbulent Kinetic Energy Scheme in the WRF-ARW Model
}

\author{
XU ZHANG \\ Shanghai Typhoon Institute, China Meteorological Administration, and Innovative Center of Regional \\ High Resolution NWP, Shanghai, China \\ JIAN-WEN BAO \\ NOAA/Earth System Research Laboratory, Boulder, Colorado \\ BAODE CHEN \\ Shanghai Typhoon Institute, China Meteorological Administration, and Innovative Center of Regional High \\ Resolution NWP, Shanghai, China \\ EVELYN D. GRELL \\ NOAA/Earth System Research Laboratory, and \\ Cooperative Institute for Research in Environmental Sciences, University of Colorado Boulder, Boulder, Colorado
}

(Manuscript received 30 November 2017, in final form 8 May 2018)

\begin{abstract}
A new three-dimensional (3D) turbulent kinetic energy (TKE) subgrid mixing scheme is developed using the Advanced Research version of the Weather Research and Forecasting (WRF) Model (WRFARW) to address the gray-zone problem in the parameterization of subgrid turbulent mixing. The new scheme combines the horizontal and vertical subgrid turbulent mixing into a single energetically consistent framework, in contrast to the conventionally separate treatment of the vertical and horizontal mixing. The new scheme is self-adaptive to the grid-size change between the large-eddy simulation (LES) and mesoscale limits. A series of dry convective boundary layer (CBL) idealized simulations are carried out to compare the performance of the new scheme and the conventional treatment of subgrid mixing to the WRF-ARW LES dataset. The importance of including the nonlocal component in the vertical buoyancy specification in the newly developed general TKE-based scheme is illustrated in the comparison. The improvements of the new scheme with the conventional treatment of subgrid mixing across the gray-zone model resolutions are demonstrated through the partitioning of the total vertical flux profiles. Results from real-case simulations show the feasibility of using the new scheme in the WRF Model in lieu of the conventional treatment of subgrid mixing.
\end{abstract}

\section{Introduction}

Historically, based on model grid size $\Delta$ and the energycontaining turbulence scale $l_{e}$, two approaches for modeling atmospheric turbulence were developed. In mesoscale numerical weather prediction (NWP) or general circulation models (GCMs), $\Delta \gg l_{e}$, which is referred to as the mesoscale limit in this study. In this limit, one-dimensional (1D) planetary boundary layer (PBL) schemes are usually used to represent subgrid-scale (SGS) turbulent motions.

\footnotetext{
Corresponding author: Xu Zhang, zhangx@typhoon.org.cn
}

The other approach for simulating atmospheric turbulence is the three-dimensional (3D) large-eddy simulation (LES), where $\Delta \ll l_{e}$ (which is referred to as the LES limit). In the LES limit, the most widely used LES SGS models include the 3D Smagorinsky-Lilly (Smagorinsky 1963; Lilly 1967) and Deardorff (1980) 3D TKE-based models (3DTKE).

As the resolution of NWP models steadily increases, such that grid size becomes comparable to the typical size of the energy-containing eddies $\left(\Delta \sim l_{e}\right)$, the grid sizes fall within a "terra incognita" (Wyngaard 2004) that is also referred to as the gray zone. In this regime, 
neither traditional PBL schemes that are designed to parameterize all PBL turbulence, nor LES SGS models that are designed to resolve the energy-containing eddies, perform appropriately.

Bridging the gap between the LES and mesoscale limits presents a number of challenges. Wyngaard (2004) provided a clear theoretical framework to unify and guide the simulation of the subgrid turbulent mixing in both limits. He proposed a unified closure concept transitioning between the LES and mesoscale limit. Honnert et al. (2011) estimated the subgrid/resolved partitioning of several turbulence variables over a large range of scales, and provided a reference for evaluating and designing the parameterization schemes in the gray zone. Shin and Hong (2015) replaced the traditional nonlocal term (Troen and Mahrt 1986; Noh et al. 2003) in the Yonsei University (YSU) model (Hong et al. 2006) with a prescribed nonlocal heat flux profile that is diagnosed from LES data, multiplying the corresponding gridsize dependency functions with the local and nonlocal profiles, to allow application of the YSU scheme to the gray zone. Ito et al. (2015) simply weighted the mesoscale dissipation length scale and horizontal diffusion with an empirical function to extend the Mellor-Yamada (MY) level-3 scheme to the gray zone. They did not use the blending method of mixing length and horizontal diffusion to merge the LES and mesoscale limits. The above two studies modified the conventional PBL parameterizations for the gray zone, but might be inappropriate for the LES limit.

Boutle et al. (2014) used a simple weighting function to blend a conventional 1D nonlocal PBL parameterization with the 3D Smagorinsky-Lilly model, based on the results of Honnert et al. (2011). The blending scheme is scale dependent, allowing a single parameterization to be used across resolutions, including the LES and mesoscale limits. With this blending scheme, they produced the seamless simulation of stratocumulus at any scale across the gray zone. Efstathiou and Beare (2015) explored the 3D Smagorinsky-Lilly SGS model beyond the LES limit. By bounding the vertical diffusion to its effective values, they extended the 3D Smagorinsky-Lilly LES model across the gray zone to the mesoscale limit. All the schemes developed in the above studies are based on the 3D Smagorinsky-Lilly model.

On the other hand, the 3DTKE SGS model (Deardorff's model) has been frequently used for representing the subgrid mixing in cloud-resolving models at kilometer grid size (Klemp and Wilhelmson 1978; Bryan et al. 2003). Parodi and Tanelli (2010) found that the LES turbulence closure "upscaled" to the gray zone is best suited to simulate deep convection. However, there are still concerns about the appropriateness of using the LES closures with mesoscale grid sizes. Bryan et al. (2003) suggested that since the LES closures were originally designed for grid sizes in the inertial subrange, the LES closures cannot be used to simulate faithfully deep moist convection with mesoscale grid sizes. They pointed out that using the LES closure is not suitable for simulations with grid size of order $1 \mathrm{~km}$. Kurowski and Teixeira (2018) proposed a pragmatic scale-adaptive TKE closure to merge the LES and mesoscale limits using a scale-adaptive mixinglength formulation. However, they only applied the eddy-diffusivity closure without considering the nonlocal term and its scale adaptivity.

The objective of this study is to develop a unified scale-adaptive 3D TKE-based closure that can be used across resolutions, including the completely unresolved/resolved extremes (LES and mesoscale limits). We propose to extend the original 3DTKE model (Deardorff 1980) that is usually used as an LES SGS model to the mesoscale limit in the framework of the Advanced Research version of the Weather Research and Forecasting (WRF) Model (WRF-ARW). Further, we develop a method to modify the 3DTKE model to make it appropriate for the range of scales between LES and mesoscale limits. As a test of the method, the modified 3DTKE model is applied to both idealized and real-data cases. A comparison with LES results and with output from several PBL parameterizations is used for evaluation. It should be noted that the present study focuses on the gray zone problem of the dry convective boundary layer (CBL).

This paper is organized as follows: section 2 gives a review of the governing equations of SGS turbulence at all scales, and provides the theoretical background for simulating the SGS turbulence in the mesoscale and LES limits. Section 3 describes the benchmark LES and the coarse-graining method. Section 4 presents the methodology for the extension to the original LES 3DTKE model, including the determinations of nonlocal flux and master length scale, and implementation of horizontal diffusion. Having developed a new 3DTKE scheme, the performance of the new scheme was evaluated by comparing various resultant profiles from conventional 1D TKE-based PBL schemes against the LES benchmark dataset for the idealized CBL case, presented in section 5. In addition, realcase simulations were carried out to test the newly developed scheme; results are presented in section 6 . Section 7 gives the conclusions along with further discussion. 


\section{Governing equations of subgrid-scale turbulence at all scales}

In this section, it is shown that the simulations of SGS mixing in the LES and mesoscale limits actually are based on the same set of governing equations, except for different closures and assumptions in the respective limits. The following discussion will provide the theoretical foundation for developing a single SGS turbulence model running in both limits.

Lilly (1967) derived the transport equations for SGS Reynolds stresses and heat fluxes, which are generally valid mathematically for both LES and mesoscale modeling. Solutions to the closure (or parameterization) problem of these equations essentially depend on the grid size of the numerical model. To facilitate the closure to these equations, Mellor (1973) and Mellor and Yamada (1982) proposed the level-4 model using closure assumptions for unknown second- and third-order terms. Following Mellor and Yamada (1974), the level-4 model is then simplified to a level-3 model, in which the differential equations for SGS stress and flux are reduced to algebraic equations. For the sake of simplicity in discussion, the algebraic equation of the deviatoric SGS stress $(i \neq j)$ in the level-3 model is considered to be

$$
\begin{aligned}
\overline{u_{i}^{\prime} u_{j}^{\prime}}= & -\frac{3 l_{1}}{\sqrt{2 e}}\left[\left(\overline{u_{i}^{\prime} u_{k}^{\prime}}-2 C_{1} e \delta_{k i}\right) \frac{\partial \bar{u}_{j}}{\partial x_{k}}\right. \\
& \left.+\left(\overline{u_{k}^{\prime} u_{j}^{\prime}}-2 C_{1} e \delta_{k j}\right) \frac{\partial \bar{u}_{i}}{\partial x_{k}}-\frac{g}{\overline{\theta_{v}}}\left(\delta_{j 3} \overline{u_{i}^{\prime} \theta_{v}^{\prime}}+\delta_{i 3} \overline{u_{j}^{\prime} \theta_{v}^{\prime}}\right)\right] .
\end{aligned}
$$

The algebraic equation of the SGS heat flux in the level3 model is written as

$\overline{u_{i}^{\prime} \theta^{\prime}}=-\frac{3 l_{2}}{\sqrt{2 e}}\left(\overline{\theta^{\prime} u_{j}^{\prime}} \frac{\partial \bar{u}_{i}}{\partial x_{j}}+\overline{u_{i}^{\prime} u_{j}^{\prime}} \frac{\partial \bar{\theta}}{\partial x_{j}}-\delta_{i 3} \frac{g}{\overline{\theta_{v}}} \overline{\theta^{\prime} \theta_{v}^{\prime}}\right)$.

Here, $u_{i}$ are the velocity components $(i=1,2,3), \theta_{v}$ is the virtual potential temperature, and $g$ is gravity. The overbars denote the quantities that are resolved by a numerical model, and primes denote deviations from the resolved quantities. The terms $l_{1}$ and $l_{2}$ are length scales that are proportional to the master mixing length scale $l, C_{1}$ is a nondimensional constant, and $e=\overline{u_{i}^{\prime 2}} / 2$ is the turbulent kinetic energy. The first two terms on the right-hand sides of Eqs. (1) and (2) are the flux productions by the local gradient of the mean state, while the third term defines the flux production by buoyancy.

In the $\mathrm{CBL}$, coherent structures due to buoyancy dominate the vertical turbulent flux in a nonlocal way
(Shin and Hong 2013; Hellsten and Zilitinkevich 2013). The flux production by buoyancy in Eqs. (1) and (2) can be regarded as the nonlocal effect. From the perspective of local and nonlocal components, Eqs. (1) and (2) can be expressed by conventional eddy-diffusivity models including the nonlocal effects as follows:

$$
\begin{aligned}
& \overline{u_{i}^{\prime} u_{j}^{\prime}}=-K_{i j}^{M}\left(\frac{\partial \bar{u}_{i}}{\partial x_{j}}+\frac{\partial \bar{u}_{j}}{\partial x_{i}}\right)+\overline{u_{i}^{\prime} u_{j}^{N L}}, \\
& \overline{u_{i}^{\prime} \theta^{\prime}}=-K_{i j}^{H} \frac{\partial \bar{\theta}}{\partial x_{j}}+\delta_{i 3} \overline{\bar{u}_{i}^{\prime} \theta^{\prime}} \mathrm{NL},
\end{aligned}
$$

where superscripts $M, H$, and NL indicate momentum, heat, and nonlocal, respectively. Thus, the flux can be decomposed into two components: the local flux due to the gradient of mean fields and the nonlocal flux due to the buoyant-production terms. Note that the nonlocal terms are only retained in the vertical. The neglect of the production by the tilting caused by the mean velocity gradient in Eq. (4) may result in the underestimation of SGS flux (Wyngaard 2004). In the level-3 model, the nonlocal effect is directly related to the buoyancy. Wyngaard (2004) emphasized that the eddy diffusivity is a second-order tensor rather than a scalar.

Wyngaard (2004) assessed the importance of buoyantproduction terms (nonlocal terms) at different grid sizes. The relative importance of the buoyancy production term (nonlocal effect) decreases with increasing $l_{e} / \Delta$, that is, in the mesoscale limit $\left(\Delta \gg l_{e}\right)$ the buoyant-production term is very important, while in the LES limit $\left(\Delta \ll l_{e}\right)$, the direct effects of buoyancy on the SGS turbulence budgets are small.

On the other hand, the SGS turbulent closures typically used in mesoscale models and LES are of the same scaling expression with $K \sim l e^{1 / 2}$ and the TKE dissipation rate $\varepsilon \sim e^{3 / 2} / l$, with the master mixing length $l$ taken as $l_{\text {Meso }}$ in mesoscale models and $l_{\text {LES }}$ in LES. It is very important to represent the master mixing length in both limits. The mixing length expression is one of the main differences between the LES and mesoscale simulation closures.

\section{a. LES limit}

The LES limit implies two assumptions: 1) the grid size is well within the inertial subrange, and 2) the grid size is much smaller than the scale of the energycontaining eddies to be simulated $\left(\Delta \ll l_{e}\right)$ (Bryan et al. 2003). Following Wyngaard (2004), the buoyancy production terms in Eqs. (3) and (4) are neglected. That is, the nonlocal mixing is explicitly calculated, and only 
small-scale eddies are parameterized, thus the local eddy-diffusivity formulation is suitable for the LES closures.

If all production terms (including the buoyancy production term) except those involving the temperature gradient in the direction of the flux are dropped, the level-3 equation for SGS heat flux [Eq. (4)] is reduced to the standard eddy-diffusivity model in the LES limit:

$$
\overline{u_{i}^{\prime} \theta^{\prime}}=-K_{H} \partial \bar{\theta} / \partial x_{i}
$$

The level-3 equation for deviatoric SGS stress [Eq. (3)] is also reduced to the eddy-diffusivity model as follows:

$$
\overline{u_{i}^{\prime} u_{j}^{\prime}}=-K_{M}\left(\partial \bar{u}_{i} / \partial x_{j}+\partial \bar{u}_{j} / \partial x_{i}\right) \quad(i \neq j) .
$$

The most widely used SGS models in LES include the 3D Smagorinsky-Lilly model and Deardorff's 3DTKE model. In the 3DTKE model, a full 3D TKE equation is solved:

$\frac{\partial e}{\partial t}=\bar{u}_{j} \frac{\partial e}{\partial x_{j}}-\overline{u_{i}^{\prime} u_{j}^{\prime}} \frac{\partial \bar{u}_{i}}{\partial x_{j}}+\frac{g}{\overline{\theta_{0}}} \overline{w^{\prime} \theta^{\prime}}-\frac{\partial \overline{u_{i}^{\prime}\left(e+p^{\prime} / \rho_{0}\right)}}{\partial x_{i}}-\varepsilon$.

The turbulent and pressure transport term is usually parameterized as

$$
\overline{u_{i}^{\prime}\left(e+p^{\prime} / \rho_{0}\right)}=-2 K_{M} \frac{\partial e}{\partial x_{i}}
$$

and the TKE dissipation rate as

$$
\varepsilon=\frac{c_{\varepsilon} e^{3 / 2}}{l},
$$

where $c_{\varepsilon}$ is a dissipation coefficient, $K_{M}=c_{k} l e^{1 / 2}$ and $K_{H}=K_{M} P_{r}^{-1}, P_{r}$ is the turbulent Prandtl number, and $c_{k}$ is a dimensionless constant. The SGS stress and flux are parameterized based on Eqs. (5) and (6).

In the LES limit where the grid size lies within the inertial subrange, it is assumed that the most energetic parameterized eddies are just a little smaller than the grid size. Deardorff's mixing length is commonly applied, which is given as the minimum of the scale adjusted for stability and the grid size:

$l=l_{\mathrm{LES}}=\left\{\begin{array}{ll}\min \left[0.76 e^{1 / 2}\left|\frac{g}{\theta} \frac{\partial \theta}{\partial z}\right|^{-1 / 2}, \Delta s\right] & \text { for } N^{2}>0 \\ \Delta s & \text { for } N^{2} \leq 0\end{array}\right.$, where $\Delta s=(\Delta x \Delta y \Delta z)^{1 / 3}$, and $N$ is the Brunt-Väisälä frequency. The 3DTKE model is widely used in LES because of its adequate accuracy in representing smallscale motions (Antonelli and Rotunno 2007; Moeng et al. 2007; Catalano and Moeng 2010).

The Smagorinsky-Lilly model is designed to simulate the energy transfer from resolved to unresolved scales across an inertial subrange of locally isotropic 3D turbulence. In the Smagorinsky-Lilly model, the deviatoric or traceless component of the SGS stress $\tau_{i j}^{d}=\left(\overline{u_{i} u_{j}}-\bar{u}_{i} \bar{u}_{j}\right)-1 / 3\left(\overline{u_{k} u_{k}}-\bar{u}_{k} \bar{u}_{k}\right) \delta_{i j}$ is closed by

$$
\tau_{i j}^{d}=-K\left(\partial \bar{u}_{i} / \partial x_{j}+\partial \bar{u}_{j} / \partial x_{i}\right)=-K D_{i j},
$$

which is the same as the expression in Eq. (6). The eddy diffusivity $K$ is a function of resolved deformation. This eddy-diffusivity model was proposed by Lilly (1967) as the "simplest reasonable closure assumption" for SGS stress. The Smagorinsky-Lilly model can be viewed as a simplification of the level-3 model for SGS stress in Eq. (1) (Wyngaard 2004).

\section{b. Mesoscale limit}

In mesoscale simulations with horizontal grid sizes $\Delta$ considerably larger than the scale of energycontaining eddies $\left(\Delta \gg l_{e}\right)$, the boundary layer is assumed to be horizontally homogeneous. It is common practice in mesoscale simulations to treat the vertical and horizontal diffusions separately. Conventional 1D PBL schemes are usually used to represent vertical mixing. Horizontal diffusion is usually treated by the simple eddy-diffusivity formulation and uses the deformation-based diffusivity coefficients. For instance, in the WRF Model, horizontal diffusion is based on a 2D first-order Smagorinsky closure, which is most often used with a $1 \mathrm{D}$ PBL scheme that independently handles the vertical diffusion. Horizontal diffusion requirements are largely a function of numerical constraints such as computational stability and small-scale noise (Deardorff 1985; Jablonowski and Williamson 2011). So, physical or energetic inconsistency may exist in this implementation.

According to Wyngaard (2004), the role of nonlocal buoyancy flux becomes more important as the grid size increases. In the mesoscale limit, the model cannot maintain any turbulence and the inclusion of the nonlocal terms is necessary. Then if one makes the boundary layer approximation and retains the term involving the scalar gradient in the direction of the flux, the level-3 model of SGS stress and flux in Eqs. (3) and (4) is reduced to 


$$
\begin{aligned}
& \overline{w^{\prime} u^{\prime}}=-K_{M v} \frac{\partial \bar{u}}{\partial z}+{\overline{w^{\prime} u^{\prime}}}^{\mathrm{NL}}, \\
& \overline{w^{\prime} \theta^{\prime}}=-K_{H v} \frac{\partial \bar{\theta}}{\partial z}+\overline{w^{\prime} \theta^{\prime}},
\end{aligned}
$$

which are the popular forms in the 1D PBL scheme. The terms $K_{M v}$ and $K_{H v}$ are vertical eddy diffusivity for momentum and heat, respectively. The eddy diffusivity is usually approximated as a scalar rather than a tensor. The vertical eddy diffusivity also takes the form of $K_{M v} \sim l_{\text {Meso }} e^{1 / 2}$, where $l_{\text {Meso }}$ is the vertical mixing length in the mesoscale, and $K_{H v}=K_{M v} P_{r}^{-1}$. In the mesoscale limit, in which the grid size is much larger than the size of the energy-containing eddies and all 3D turbulent motions are unresolved, the mixing length formulation would be different from that of the LES limit. In regard to the nonlocal term, there are many definitions based on different assumptions, especially for $\overline{w^{\prime} \theta^{\prime N L}}$ (Troen and Mahrt 1986; Deardorff 1972; Holtslag and Moeng 1991; Siebesma et al. 2007; Noh et al. 2003; Shin and Hong 2015), which will be discussed later.

On the other hand, SGS horizontal fluxes can be expressed by eddy-diffusivity formulations,

$$
\begin{aligned}
& \overline{u^{\prime} v^{\prime}}=-K_{M h}\left(\frac{\partial \bar{u}}{\partial y}+\frac{\partial \bar{v}}{\partial x}\right), \\
& \overline{u^{\prime} \theta^{\prime}}=-K_{H h} \frac{\partial \bar{\theta}}{\partial x},
\end{aligned}
$$

where $K_{M h}$ and $K_{H h}$ are horizontal eddy diffusivities for momentum and heat, respectively. In the WRF Model, $K_{M h}$ and $K_{H h}$ are calculated by 2D Smagorinsky firstorder closure based on the horizontal resolvable-scale velocity deformation.

From the above discussion, the two approaches for simulating subgrid turbulent mixing (LES and mesoscale simulation) appear different. Actually, the exact but unclosed governing equations for subgrid turbulent mixing in these two approaches are mathematically identical. Historically, based on the assumption of scale separation, the conventional subgrid turbulent mixing in mesoscale models and LES implemented different closure schemes. Wyngaard (2004) conceptualized a generalized closure to unify the governing equations for subgrid mixing across mesoscale and LES limits. In support of Wyngaard's theory, we explore ways to unify the mesoscale simulations and LES in a single code that allows the transition between LES and mesoscale limits based on the 3DTKE model using the WRF framework.

As mentioned above, the SGS mixing parameterization in the mesoscale modeling mainly differs from the LES SGS model in two key aspects, including the determination of nonlocal flux (heat and momentum) and the determination of length scale suitable for the mesoscale. Therefore, after describing the benchmark LES, we will determine the nonlocal heat flux and the master mixing length suitable for the mesoscale limit based on the LES output data.

\section{The benchmark LES}

For the benchmark LES, the standard LES configuration of the WRF Model was used (Skamarock et al. 2008), and the simulation was performed over a $30 \mathrm{~km} \times 30 \mathrm{~km}$ domain with a horizontal grid size of $50 \mathrm{~m}$. The model top is at $2 \mathrm{~km}$ with 100 approximately equally spaced vertical layers. The lateral boundary conditions in the $x$ and $y$ directions are cyclic. The benchmark LES was driven by surface heat flux $\left(Q_{0}=0.24 \mathrm{~K} \mathrm{~m} \mathrm{~s}^{-1}\right.$ or about $\left.273 \mathrm{~W} \mathrm{~m}^{-2}\right)$ and geostrophic wind in the $x$ direction $\left(U_{g}=10 \mathrm{~m} \mathrm{~s}^{-1}\right)$. The 3DTKE model (Deardorff 1980) is selected for SGS turbulence parameterization. Time integration is performed with a time step of $0.2 \mathrm{~s}$ for a 4 -h period. The initial sounding of the potential temperature is

$$
\theta= \begin{cases}300 \mathrm{~K} & : 0<z \leq 925 \mathrm{~m} \\ 300 \mathrm{~K}+(z-925 \mathrm{~m}) \times 0.0536 \mathrm{Km}^{-1} & : 925<z \leq 1075 \mathrm{~m} \\ 308.05 \mathrm{~K}+(z-1075 \mathrm{~m}) \times 0.003 \mathrm{~K} \mathrm{~m}^{-1} & : z>1075 \mathrm{~m}\end{cases}
$$

The LES output was used to determine the nonlocal heat flux and evaluate the performance of the newly developed 3DTKE scheme. The coarse-graining approach was used as a tool to examine model behavior with varying grid size (Honnert et al. 2011; Shin and Hong 2015). In the coarse-graining method, successive horizontal spatial means are applied to the original LES fields to derive lower resolution fields, which are considered to be the "truth" for the lower resolution.

\section{Methodology}

Our starting point is the original 3DTKE model in the WRF-ARW, whose closures are based on Eqs. (5), (6), (8), and (9). Because Eqs. (5) and (6) represent only the 
local terms, in this section we present specific techniques for the determinations of nonlocal heat/momentum fluxes, which will be added to Eqs. (5) and (6), in order to apply these equations on the full range of scales from mesoscale to LES limits. Then, we will determine the master mixing length for the mesoscale limit, and propose algorithms for the transitioning of vertical and horizontal diffusions between LES and mesoscale limits, based on the 3DTKE model within the framework of WRF.

\section{a. Analysis and determination of nonlocal heat flux}

From the above discussion, the nonlocal effect due to buoyancy is very important in the mesoscale limit. As shown in Eq. (13), the popular format for the SGS heat flux in the mesoscale limit can be expressed as the sum of the eddy-diffusivity (local) component and nonlocal component. This subsection describes a method to determine the nonlocal component, following the methods of Shin and Hong $(2013$, 2015).

First, the mesoscale grid box in the dry CBL is considered to consist of convective updrafts and environmental air. Using the LES solution, these turbulent motions can be explicitly resolved. Therefore, the total turbulent heat flux can be decomposed into three terms (Siebesma and Cuijpers 1995; Siebesma et al. 2007):

$\overline{w^{\prime} \theta^{\prime}}=a \overline{w^{\prime} \theta^{\prime}}+(1-a) \overline{w^{\prime} \theta^{\prime}}+a(1-a)\left(w_{c}-w_{e}\right)\left(\theta_{c}-\theta_{e}\right)$,

where $a$ is the areal fraction of the convective updrafts in the grid box. The overbar indexed $c(e)$ denotes an average of the perturbations from the convective updraft (environmental) average within the areadesignated convective (environment). The subscript $c(e)$ refers to an average over the convective updraft (environmental) area. The first term is the turbulent heat flux in the convective updraft; the second describes the environmental turbulence, while the third term describes the organized turbulence term. In the present study, in the dry CBL, the first two terms can be considered to be the local heat flux due to small eddies, while the third term can be understood to be nonlocal heat flux induced by coherent structures (i.e., large eddies). Therefore, we can separate the local $(L)$ and nonlocal (NL) heat flux as

$$
\begin{aligned}
{\overline{w^{\prime} \theta^{\prime}}}^{\mathrm{NL}} & =a(1-a)\left(w_{c}-w_{e}\right)\left(\theta_{c}-\theta_{e}\right), \\
{\overline{w^{\prime} \theta^{\prime}}}^{L} & =a \overline{w^{\prime} \theta^{\prime}}+(1-a) \overline{w^{\prime} \theta^{\prime}} .
\end{aligned}
$$

The conditional sampling method of Siebesma et al. (2007) is used to identify the coherent structures and

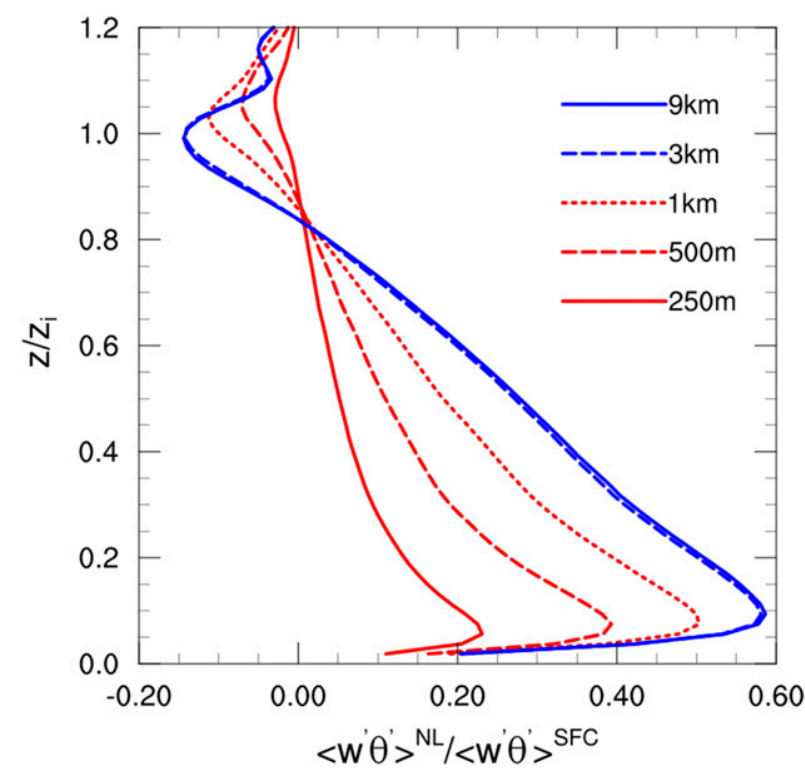

FIG. 1. Vertical profiles of horizontally averaged SGS nonlocal heat flux for different resolutions $(9 \mathrm{~km}, 3 \mathrm{~km}, 1 \mathrm{~km}, 500 \mathrm{~m}$, and $250 \mathrm{~m}$ ), normalized by the surface heat flux. The vertical axis is height $z$ normalized by the PBL depth $z_{i}$.

distinguish the nonlocal transport from the total transport, based on the magnitude of the vertical velocity:

$$
w(x, y, z)>w_{p \%}(z) .
$$

The $p$-percentile velocity $w_{p \%}(z)$ is defined as the vertical velocity for which exactly $p \%$ of the grid points exceed $w_{p} \%(z)$ at height $z$. Here, $p=15$ is used. There are many studies addressing how best to quantify the contribution of the coherent structures to the total flux (Lenschow and Stephens 1980; Greenhut and Khalsa 1982; Schmidt and Schumann 1989; Couvreux et al. 2010; Hellsten and Zilitinkevich 2013). Note that there is no unique and strict way to separate the coherent structures from the environmental turbulence as the two kinds of motion are strongly intertwined. In reality, the nonlocal transports drive the local mixing through the turbulence cascade, thus there is no spectral gap separating them.

Based on the formulations of Eqs. (18) and (19), the total SGS heat flux can be decomposed into SGS nonlocal and local heat flux at different resolution of $\Delta$ as follows:

$$
\begin{aligned}
{\overline{w^{\prime} \theta^{\prime}}}^{\Delta, \mathrm{NL}} & =a^{\Delta}\left(1-a^{\Delta}\right)\left(w_{c}^{\Delta}-w_{e}^{\Delta}\right)\left(\theta_{c}^{\Delta}-\theta_{e}^{\Delta}\right), \\
\overline{w^{\prime} \theta^{\prime}} & =a^{\Delta, L} \overline{w^{\prime} \theta^{\prime}}+, c+\left(1-a^{\Delta}\right) \overline{w^{\prime} \theta^{\prime}}+, e+\bar{f}^{\Delta},
\end{aligned}
$$




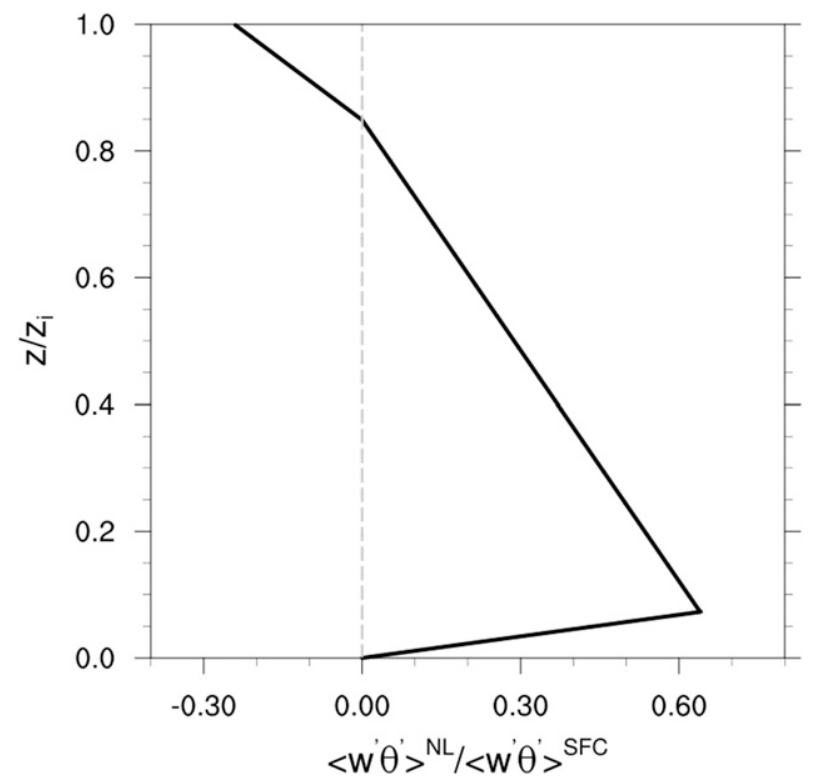

FIG. 2. The vertical profile of the prescribed total nonlocal heat flux, expressed by Eq. (23). The horizontal axis is normalized by the surface heat flux. The vertical axis is height $z$ normalized by the PBL depth $z_{i}$.

where $f$ is the SGS heat flux from the LES SGS model. By applying the coarse-graining method to the LES data, together with the conditional sampling technique, explicit solutions to the above equations can be derived.

Using Eq. (21), vertical profiles of the SGS nonlocal heat flux for different resolutions are diagnosed from the benchmark LES, and are shown in Fig. 1. The magnitudes and vertical profiles are similar to those of Hellsten and Zilitinkevich (2013) and Shin and Hong (2015). The role of the SGS nonlocal flux is to cool the surface layer and warm the mixed layer. Negative heat flux can be found in the entrainment zone. The magnitude of the SGS nonlocal heat flux is decreasing as the grid size decreases, which means that the nonlocal component is explicitly represented by the model dynamics. This implies that in the LES limit, parameterizing the turbulent flux through the eddy-diffusivity formulation is appropriate only if the LES is converged at the resolution of $50 \mathrm{~m}$, and that in the mesoscale limit, the effect of nonlocal transport is necessary to accurately represent the SGS flux. This finding is consistent with the theoretical analysis of Wyngaard (2004). As the grid size increases to the mesoscale limit, the vertical profile of the SGS nonlocal heat flux converges to a single profile (cf. profiles for 3 and $9 \mathrm{~km}$ in Fig. 1). Based on this analysis, it can be assumed that at a resolution of $9 \mathrm{~km}$, all the nonlocal heat flux is subgrid.

Following the method of Shin and Hong (2013), the vertical profile of the nonlocal heat flux is designed to fit the domain-averaged LES profile at the mesoscale limit

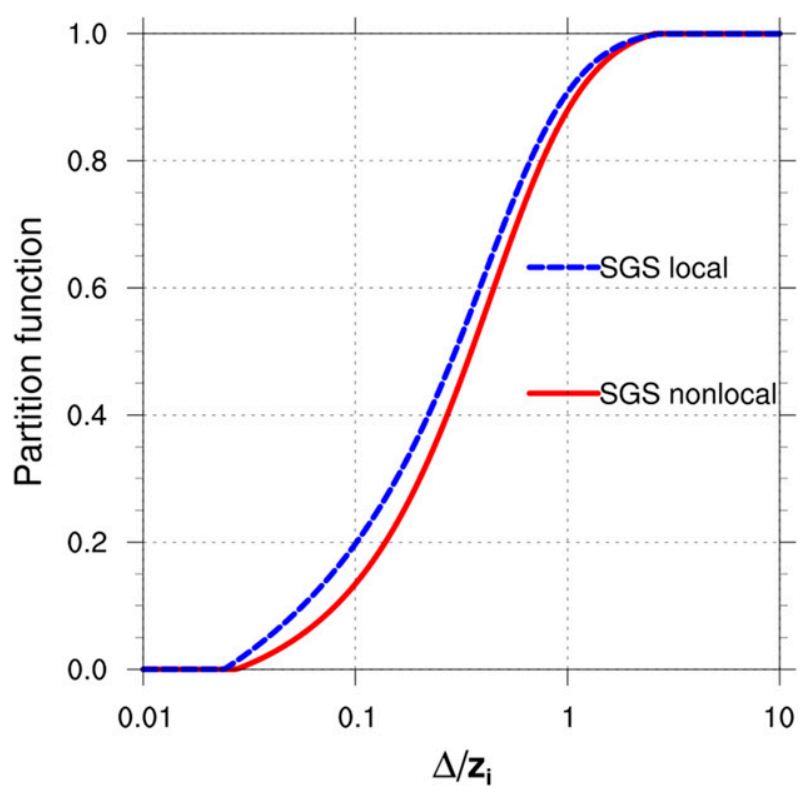

FIG. 3. Partition functions for SGS local $\left[P_{L}\left(\Delta / z_{i}\right)\right.$, (blue dashed)] and nonlocal $\left[P_{\mathrm{NL}}\left(\Delta / z_{i}\right)\right.$, (red solid)] heat flux.

(i.e., the profile at a resolution of $9 \mathrm{~km}$ ) (Fig. 2). The nonlocal heat flux consists of three parts: surface-layer cooling, mixed-layer heating, and entrainment. It is a linear function of $z_{*}\left(z_{*} \equiv z / z_{i}\right)$ :

$$
{\overline{w^{\prime} \theta^{\prime}}}^{\mathrm{NL}}=\left\{\begin{array}{l}
{\overline{w^{\prime} \theta^{\prime}}}_{\mathrm{SL}}^{\mathrm{NL}}=A_{1} z_{*}+B_{1}: 0.0 \leq z_{*} \leq \delta_{* \mathrm{SL}} \\
\overline{w^{\prime} \theta^{\prime}} \mathrm{ML}=A_{2} z_{*}+B_{2}: \delta_{* \mathrm{SL}} \leq z_{*} \leq 1.0-\delta_{* \mathrm{EZ}} \\
\overline{w^{\prime} \theta^{\prime}} \mathrm{\textrm {EZ }}=A_{3} z_{*}+B_{3}: 1.0-\delta_{* \mathrm{EZ}} \leq z_{*} \leq 1.0
\end{array}\right.
$$

where $z_{i}$ is the PBL height, which is simply defined as the lowest level at which the potential temperature is $0.5 \mathrm{~K}$ larger than that at the first model level; $\delta *_{\mathrm{SL}}$ is the thickness of the surface layer $\left(\delta *_{\mathrm{SL}}=0.08\right)$; and $\delta *_{\mathrm{EZ}} \equiv \delta_{\mathrm{EZ}} / z_{i}$, where $\delta_{\mathrm{EZ}}$ is the thickness of the entrainment zone. Solving the linear system, the coefficients $A_{1}, A_{2}, A_{3}, B_{1}, B_{2}$, and $B_{3}$ are determined as follows:

$$
\left\{\begin{array}{l}
A_{1}={\overline{w^{\prime}{ }^{\prime}}}_{\mathrm{ML}}^{\mathrm{NL}}\left(z_{*}=\delta_{*_{\mathrm{SL}}}\right) / \delta_{*_{\mathrm{SL}}} \\
B_{1}=0 \\
A_{2}=-\overline{w^{\prime} \theta_{\mathrm{ML}}^{\prime}} \mathrm{NL}\left(z_{*}=\delta_{*_{\mathrm{SL}}}\right) /\left[\left(1-\delta_{*_{\mathrm{EZ}}}\right)-\delta_{*_{\mathrm{SL}}}\right] \\
B_{2}=-\left(1-\delta_{*_{\mathrm{EZ}}}\right) A_{2} \\
A_{3}=A_{R, \mathrm{NL}} \overline{w^{\prime} \theta^{\prime}} / \delta_{\mathrm{SFC}} / \delta_{\mathrm{EZ}} \\
B_{3}=-\left(1-\delta_{*_{\mathrm{EZ}}}\right) A_{3}
\end{array}\right.
$$

According to Deardorff et al. (1980) and Noh et al. (2003), the thickness of the entrainment zone is estimated as 


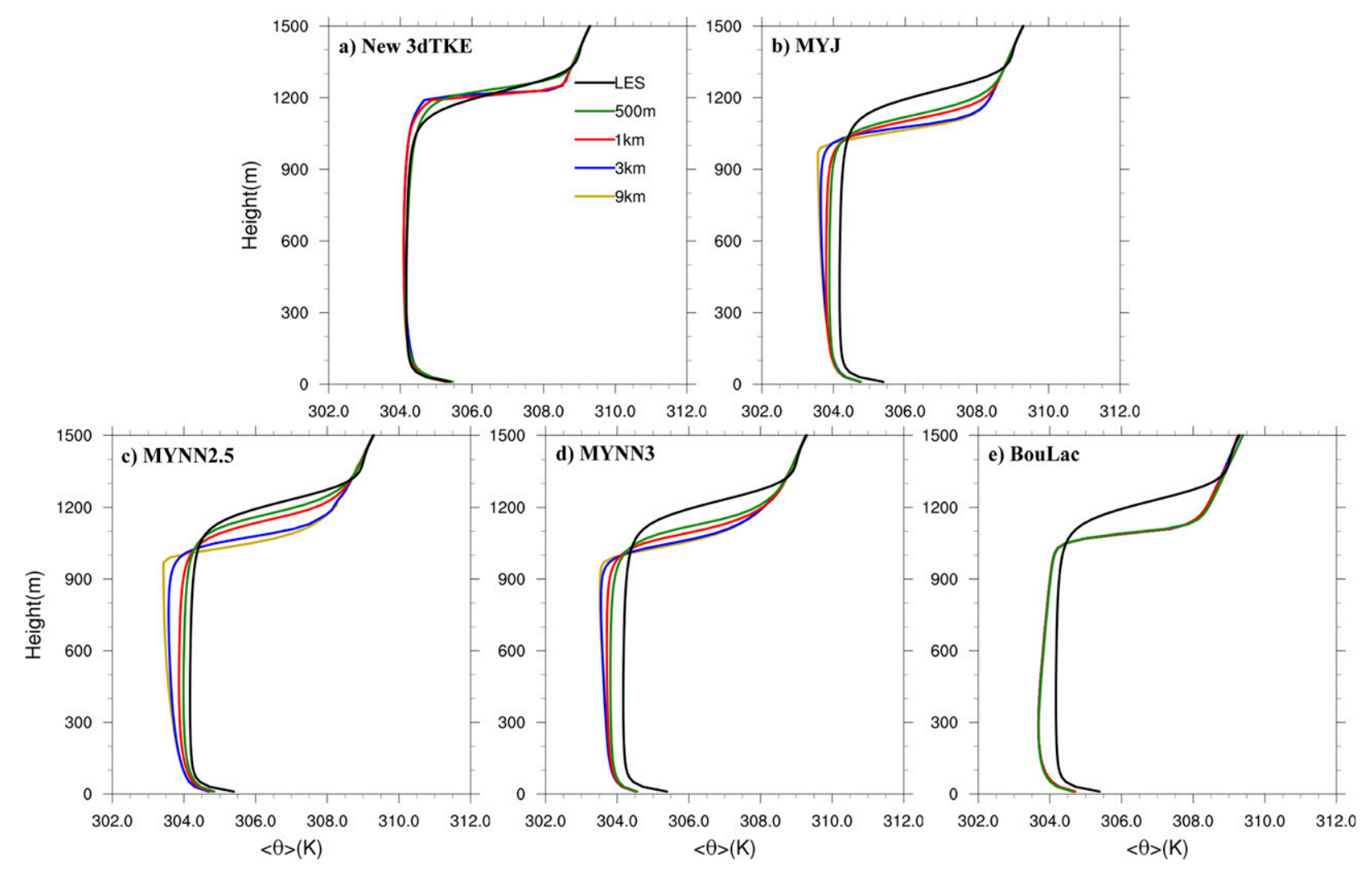

FIG. 4. Vertical profiles of horizontally averaged potential temperature for the (a) newly developed 3DTKE, (b) MYJ, (c) MYNN2.5, (d) MYNN3, and (e) BouLac schemes, at different resolutions $(9 \mathrm{~km}, 3 \mathrm{~km}, 1 \mathrm{~km}$, and $500 \mathrm{~m})$ at $4 \mathrm{~h}$. The heavy black line shows the result from the benchmark LES run.

$\delta_{\mathrm{EZ}} / z_{i}=d_{1}+d_{2} \mathrm{Ri}_{*}^{-1}$. The empirical constants $d_{1}=0.02$ and $d_{2}=0.05$ are used. The term $\mathrm{Ri}_{*}=\left(g / \theta_{0}\right) z_{i} \Delta \theta / w_{m}^{2}$ is the convective Richardson number. Here $\Delta \theta$ is the change of potential temperature across the inversion layer, and $w_{m}$ is a velocity scale that considers both buoyancy and surface-shear effects (Moeng and Sullivan 1994): $w_{m}^{3}=w_{*}^{3}+5 u_{*}^{3} ; w_{*}$ is the convective velocity, and $u_{*}$ the surface friction velocity.

Lenschow (1974) suggested that $\overline{w^{\prime} \theta^{\prime}}{ }_{\mathrm{ML}}\left(z_{*}\right)=$ $\left(1-1.15 z_{*}\right) \overline{w^{\prime} \theta^{\prime}}$ SFC for $0<z_{*} \leq \underline{0.87}$. The ratio of nonlocal heat flux to total heat flux $\overline{w^{\prime} \theta^{\prime}} \mathrm{ML}\left(z_{*}\right) / \overline{w^{\prime} \theta^{\prime}}$ ML $\left(z_{*}\right)$ is set to 0.65 based on the LES data analysis. The variable $A_{R, \mathrm{NL}}$ is the ratio of nonlocal entrainment flux to the surface flux $\left[A_{R, \mathrm{NL}} \equiv \overline{w^{\prime} \theta^{\prime}} \mathrm{EZ}^{\mathrm{LL}}\left(z_{*}=1.0\right) / \overline{w^{\prime} \theta^{\prime}} \mathrm{SFC}\right]$, and can be estimated as $A_{R, \mathrm{NL}}=f_{\text {ent }} A_{R}$, where the empirical constant $f_{\text {ent }}=2.0$ is used here. Suggested by Conzemius and Fedorovich (2006), the ratio of entrainment flux to the surface flux $A_{R}\left[A_{R} \equiv \overline{w^{\prime} \theta^{\prime}}\left(z_{*}=1.0\right) / \overline{w^{\prime} \theta^{\prime}}\right.$ SFC $]$ is estimated as

$$
A_{R}=\frac{w_{m}^{3}}{w_{*}^{3}} \frac{0.2}{1-0.4 \mathrm{Ri}_{\mathrm{GS}}^{-1}} .
$$

The quantity $\mathrm{Ri}_{\mathrm{GS}}$ is the Richardson number associated with the entrainment zone shear.

\section{b. Determination of nonlocal momentum flux}

Following the suggestion by Brown and Grant (1997) and Noh et al. (2003), the effect of nonlocal momentum flux is included in the momentum flux profile and added only to the vertical component of the 3D SGS flux [Eq. (6)]:

$$
\overline{u^{\prime} w^{\prime}}=-K_{M v}\left(\frac{\partial \bar{u}}{\partial z}+\frac{\partial \bar{w}}{\partial x}\right)+K_{M v} \gamma_{m}
$$

The countergradient term $\gamma_{m}$ of momentum flux is given as

$$
\gamma_{m}=-S_{m} \frac{u_{*}^{2}}{w_{s} z_{i}}\left(\frac{w_{*}}{w_{s}}\right)^{3}
$$

where the velocity scale $w_{s}=\left(u_{*}^{3}+8 k w_{*}^{3} z / z_{i}\right)^{1 / 3}$. The empirical constant $S_{m}=15.9$ is used following Noh et al. (2003).

c. Determination of the vertical master mixing length $l_{v}$

To extend the 3DTKE model to the mesoscale limit, the turbulent length scale in the boundary layer should 

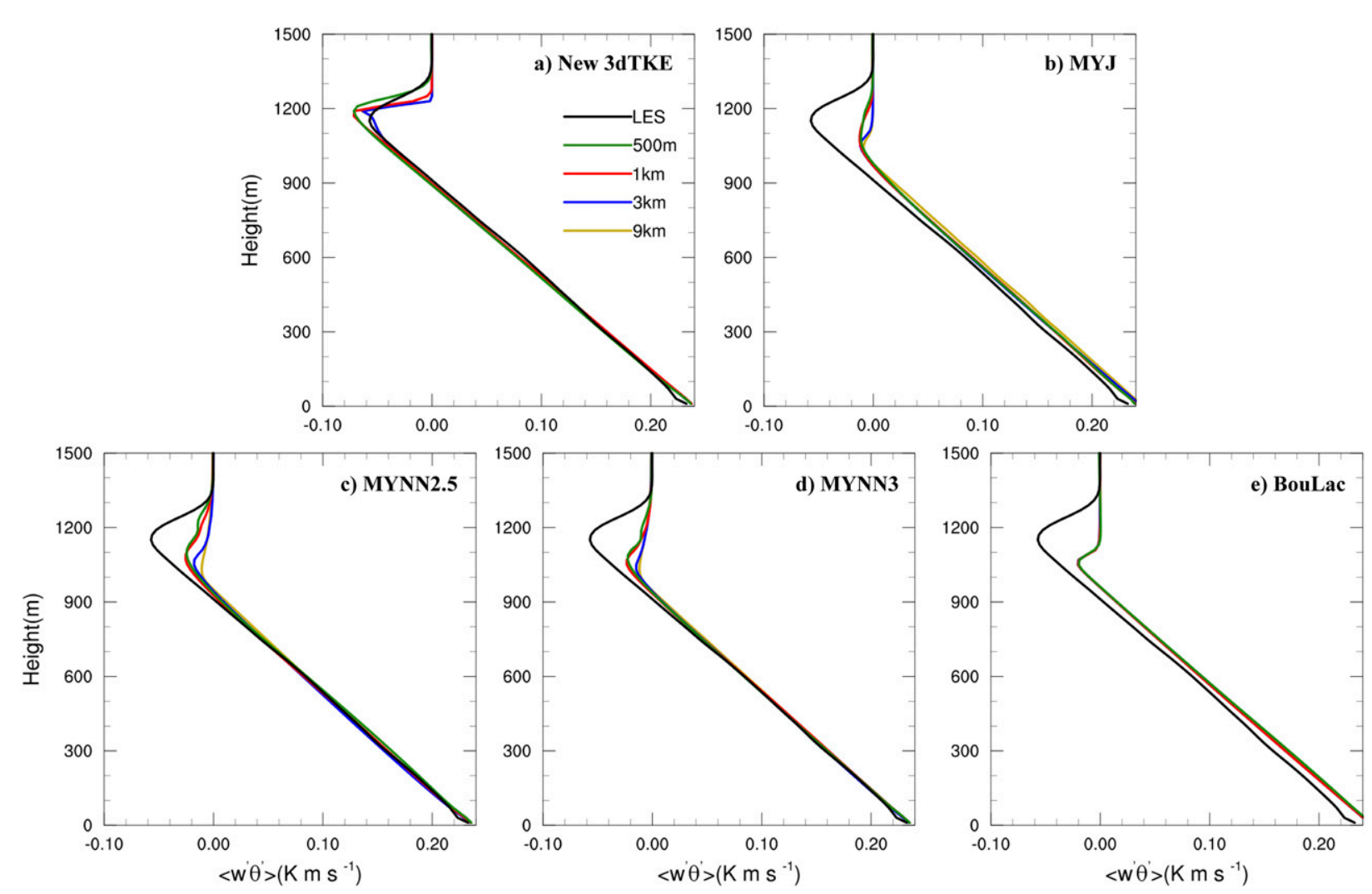

FIG. 5. As in Fig. 4, but for the total vertical heat flux.

be highly correlated with the distance from the ground, structure and strength of the turbulence, and local thermal stability. Considering these effects, the length scale formulation of the MellorYamada level-3 (MYNN) scheme (Nakanishi and Niino 2009) is chosen to be used in the new scheme, in which the vertical master mixing length $l_{v}\left(l_{\text {Meso }}\right)$ is determined by a harmonic average of three length scales as

$$
\frac{1}{l_{v}}=\frac{1}{l_{\text {Meso }}}=\frac{1}{L_{S}}+\frac{1}{L_{T}}+\frac{1}{L_{B}},
$$

where $L_{S}$ is the mixing length in the surface layer controlled by the effects of wall and stability, $L_{T}$ is the mixing length depending on the turbulent structure of the PBL (Mellor and Yamada 1982), and $L_{B}$ the mixing length limited by the thermal stability. The $L_{S}, L_{T}$, and $L_{B}$ are given by

$$
\begin{aligned}
& L_{S}=\alpha_{1} k z \\
& L_{T}=\alpha_{2} \frac{\int_{0}^{\infty} e^{1 / 2} z d z}{\int_{0}^{\infty} e^{1 / 2} d z},
\end{aligned}
$$

$$
L_{B}=\left\{\begin{array}{ll}
{\left[\alpha_{3} e^{1 / 2}+\alpha_{4} e^{1 / 2}\left(q_{c} / L_{T} N\right)^{1 / 2}\right] / N,} & \partial \bar{\theta} / \partial z>0 \\
\infty, & \partial \bar{\theta} / \partial z \leq 0
\end{array} .\right.
$$

In addition to the above length scale, a minimum limit of mixing length is defined by

$$
L_{f}=\left\{\begin{array}{ll}
\alpha_{3} e^{1 / 2} / N, & \partial \bar{\theta} / \partial z>0 \\
\infty, & \partial \bar{\theta} / \partial z \leq 0
\end{array} .\right.
$$

Finally, the vertical master mixing length is determined by

$$
l_{\text {Meso }}=\min \left[L_{B} /\left(L_{B} / L_{S}+L_{B} / L_{T}+1\right), L_{f}\right],
$$

where $\alpha_{1}, \alpha_{2}, \alpha_{3}$, and $\alpha_{4}$ are empirical constants; $k$ is the von Kármán constant; $N$ is the Brunt-Väisälä frequency; and $q_{c} \equiv\left[\left(g / \theta_{0}\right) Q_{0} L_{T}\right]^{1 / 3}$ is a velocity scale similar to the convective velocity $w_{*}$. Note that the $L_{S}$ formulation is different from that of the MYNN level-3 scheme; a simplified expression is used in the present study. In this study, $\alpha_{1}=1.9$, $\alpha_{2}=0.9, \alpha_{3}=1.0$, and $\alpha_{4}=5$ are set by matching the heat flux profile with LES data. Note that this method of calculating the mixing length is only in the vertical. The implementation of horizontal diffusion will be discussed later. 


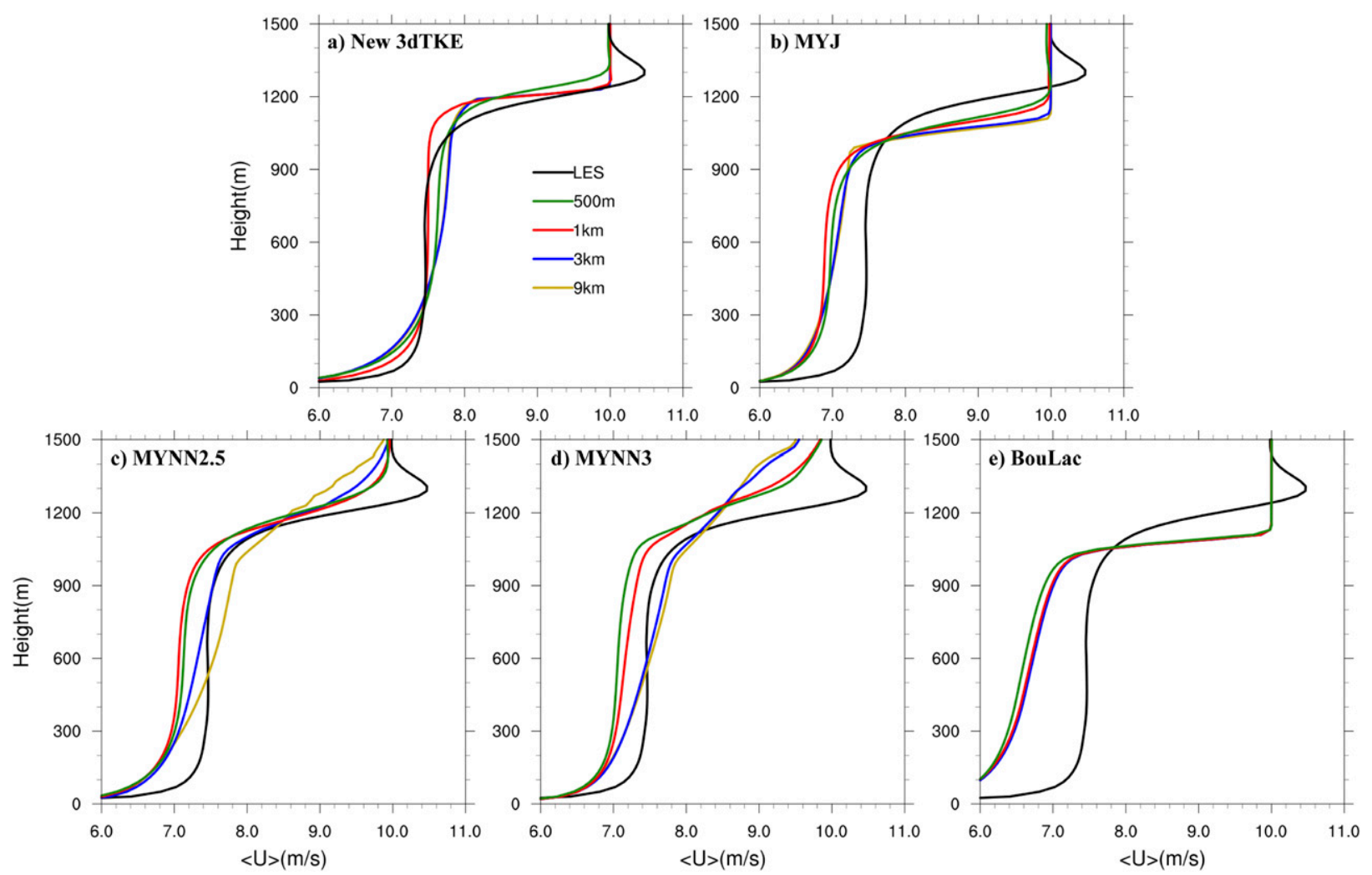

FIG. 6. As in Fig. 4, but for the $u$ component of mean wind.

\section{d. Transition between the LES and mesoscale limits}

The SGS heat flux for a particular grid size $\Delta$ can be divided into the local and nonlocal components:

${\overline{w^{\prime} \theta^{\prime}}}^{\Delta}=-K_{H v}^{\Delta} \frac{\partial \bar{\theta}}{\partial z}+{\overline{w^{\prime} \theta^{\prime}}}^{\Delta, \mathrm{NL}}={\overline{w^{\prime} \theta^{\prime}}}^{\Delta, L}+{\overline{w^{\prime} \theta^{\prime}}}^{\Delta, \mathrm{NL}}$,

where the subscript $v$ refers to vertical. Because higherresolution simulations better resolve turbulent heat flux and the large eddies responsible for nonlocal heat flux, the SGS nonlocal flux term is downweighted by the partition function $P_{\mathrm{NL}}\left(\Delta / z_{i}\right)$ :

$$
{\overline{w^{\prime} \theta^{\prime}}}^{\Delta, \mathrm{NL}}={\overline{w^{\prime} \theta^{\prime}}}^{\mathrm{NL}} P_{\mathrm{NL}}\left(\Delta / z_{i}\right)
$$

where the total SGS nonlocal heat flux ${\overline{w^{\prime} \theta^{\prime}}}^{\mathrm{NL}}$ is determined by Eq. (23). In the LES limit, $P_{\mathrm{NL}}\left(\Delta / z_{i}\right)=0$, so there is no nonlocal heat flux, and Eq. (34) becomes the eddy-diffusivity formulation, which is commonly used in the LES closure. For $\Delta \gg z_{i}, P_{\mathrm{NL}}\left(\Delta / z_{i}\right)=1$ and the entire nonlocal term is retained. The calculations of the partition functions are given in the appendix, and the resulting curves are shown in Fig. 3.

The SGS local heat flux is parameterized using the eddy-diffusivity formulation, and also decreases as grid size decreases. The vertical heat eddy diffusivities for $\Delta$ are written as $K_{H v}^{\Delta}=L_{\Delta} e^{1 / 2}$, thus the SGS local heat flux for $\Delta$ can be expressed by

$$
{\overline{w^{\prime} \theta^{\prime}}}^{\Delta, L}=-K_{H v}^{\Delta} \frac{\partial \bar{\theta}^{\Delta}}{\partial z}=-L_{\Delta} e^{1 / 2} \frac{\partial \bar{\theta}^{\Delta}}{\partial z}
$$

The length scale $L_{\Delta}$ is obtained by blending the LES length scale $L_{\mathrm{LES}}=c_{k 1} l_{\mathrm{LES}}$ and the mesoscale length scale $L_{\text {Meso }}=c_{k 2} l_{\text {Meso }}$, weighted by the partition function $P_{L}\left(\Delta / z_{i}\right)$ for local heat flux, where $c_{k 1}$ and $c_{k 2}$ are dimensionless constants:

$$
L_{\Delta}=P_{L}\left(\Delta / z_{i}\right) L_{\text {Meso }}+\left[1-P_{L}\left(\Delta / z_{i}\right)\right] L_{\text {LES }} .
$$

Therefore, when at the mesoscale limit $P_{\mathrm{NL}}\left(\Delta / z_{i}\right)=1$ and $P_{L}\left(\Delta / z_{i}\right)=1$ (Fig. 3), the mixing length scale $L_{\text {Meso }}$ and the nonlocal term $\bar{w}^{\prime} \theta^{\prime N L}$ are used to make the model suitable for the mesoscale, while at the LES limit $P_{\mathrm{NL}}\left(\Delta / z_{i}\right)=0$ and $P_{L}\left(\Delta / z_{i}\right)=0$ (Fig. 3), the original Deardorff's 3DTKE model is recovered. Note that the dimensionless constants $c_{k 1}$ and $c_{k 2}$ are different at LES and mesoscale limits; in this study $c_{k 1}=$ 0.1 and $c_{k 2}=0.4$ give the best results. The methods of blending the mixing length and downweighting the 

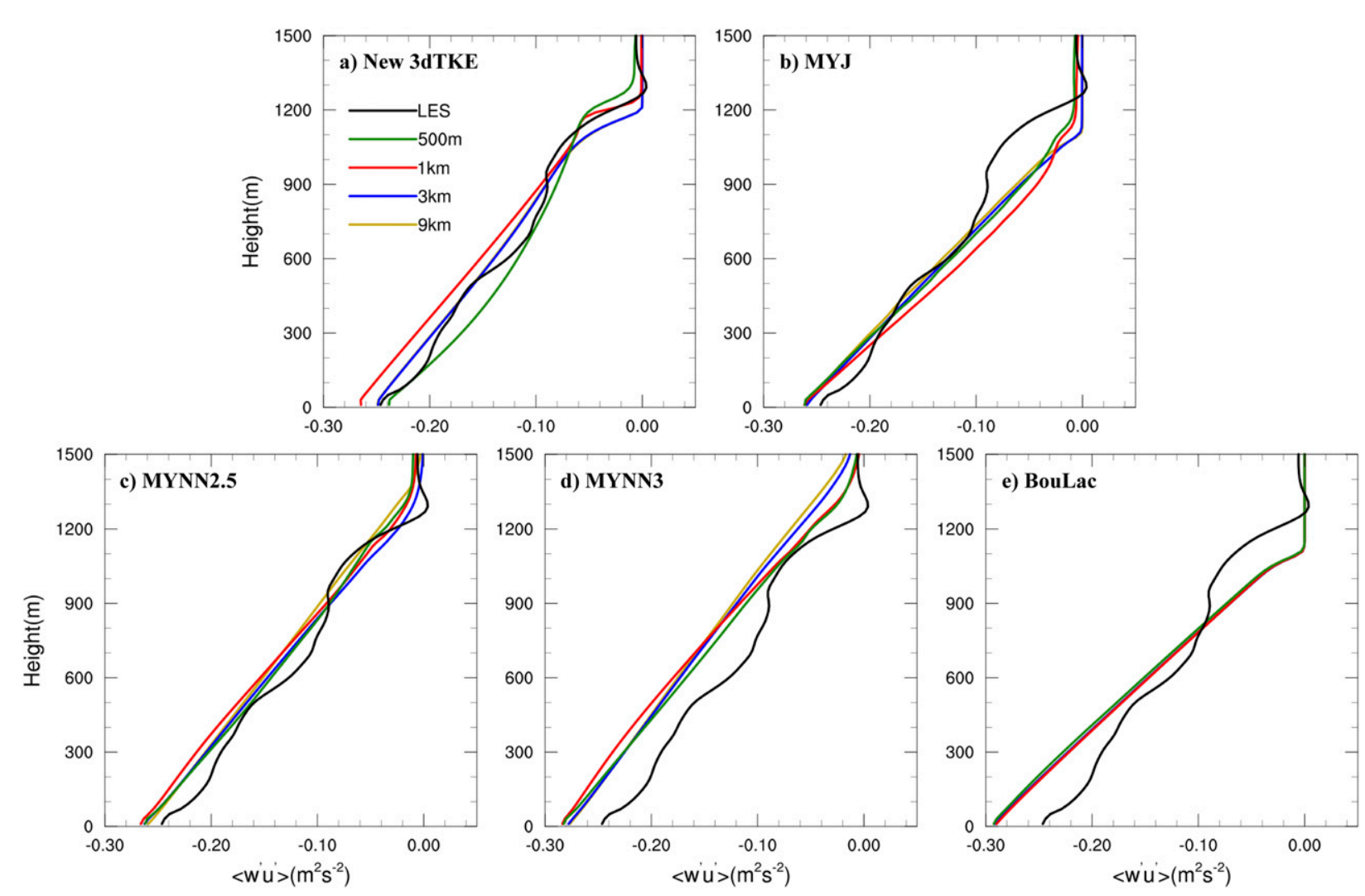

FIG. 7. As in Fig. 4, but for the mean vertical momentum flux.

nonlocal term are from Boutle et al. (2014) [see their Eqs. (2) and (4)].

In terms of the dissipation rate for different $\Delta$ in the TKE equation,

$$
\varepsilon_{\Delta}=\frac{e^{3 / 2}}{L_{\varepsilon \Delta}},
$$

the dissipation length scale $L_{\varepsilon \Delta}$ is determined by blending the LES length scale $L_{\varepsilon \mathrm{LES}}=c_{\varepsilon 1} l_{\mathrm{LES}}$ and the mesoscale length scale $L_{\varepsilon \text { Meso }}=c_{\varepsilon 2} l_{\text {Meso }}$ using the partition function $P_{\mathrm{TKE}}\left(\Delta / z_{i}\right)$ :

$$
L_{\varepsilon \Delta}=P_{\mathrm{TKE}}\left(\Delta / z_{i}\right) L_{\varepsilon \text { Meso }}+\left[1-P_{\mathrm{TKE}}\left(\Delta / z_{i}\right)\right] L_{\varepsilon \mathrm{LES}} .
$$

The dissipation coefficient for LES, $c_{\varepsilon 1}=1 /(0.19+$ $0.74 l_{\text {LES }} / \Delta s$ ), is the typical value used in Deardorff's original 3DTKE model (Deardorff 1980). In the mesoscale limit, the dissipation coefficient $c_{\varepsilon 2}=3.5$ is used in this study. The TKE dissipation rate approaches the LES limit as grid size decreases. In addition, the scale dependency of the SGS momentum flux is accomplished by multiplying the partition function for TKE, $P_{\mathrm{TKE}}\left(\Delta / z_{i}\right)$, with the countergradient term [Eq. (27)].

\section{e. Implementation of horizontal diffusion}

In the new 3DTKE scheme, a pragmatic blending approach for the transition of horizontal diffusion across the gray zone is introduced as

$$
\begin{aligned}
K_{h}= & K_{D}+K_{T}=P_{L}\left(\Delta x / z_{i}\right)\left(c_{s} l_{h}\right)^{2} D / \sqrt{2} \\
& +\left[1-P_{L}\left(\Delta x / z_{i}\right)\right] c_{k} l_{h} e^{1 / 2},
\end{aligned}
$$

where $K_{D}$ is the diffusivity based on the deformation (i.e., Smagorinsky-type formula), $K_{T}$ is the diffusivity based on the TKE, and $c_{s}$ and $c_{k}$ are dimensionless constants. The horizontal mixing length is defined as $l_{h}=(\Delta x \Delta y)^{1 / 2}$, and $D=\left(D_{i j} D_{i j}\right)^{1 / 2}$.

Using the partition function $P_{L}\left(\Delta / z_{i}\right)$, this approach blends the Smagorinsky-Lilly closure based on the deformation for mesoscale simulation, with the TKEbased subgrid mixing scheme for LES. When at the mesoscale limit $P_{L}\left(\Delta / z_{i}\right)=1$ (Fig. 3), and the horizontal diffusivity based on the Smagorinsky-Lilly formula is obtained. At the LES limit, $P_{L}\left(\Delta / z_{i}\right)=0$, and the horizontal eddy diffusivity is calculated by TKE, which retains the formulation in the original 3DTKE model (consistent with the vertical eddy diffusivity). Analogous approaches that include the TKE term in the 

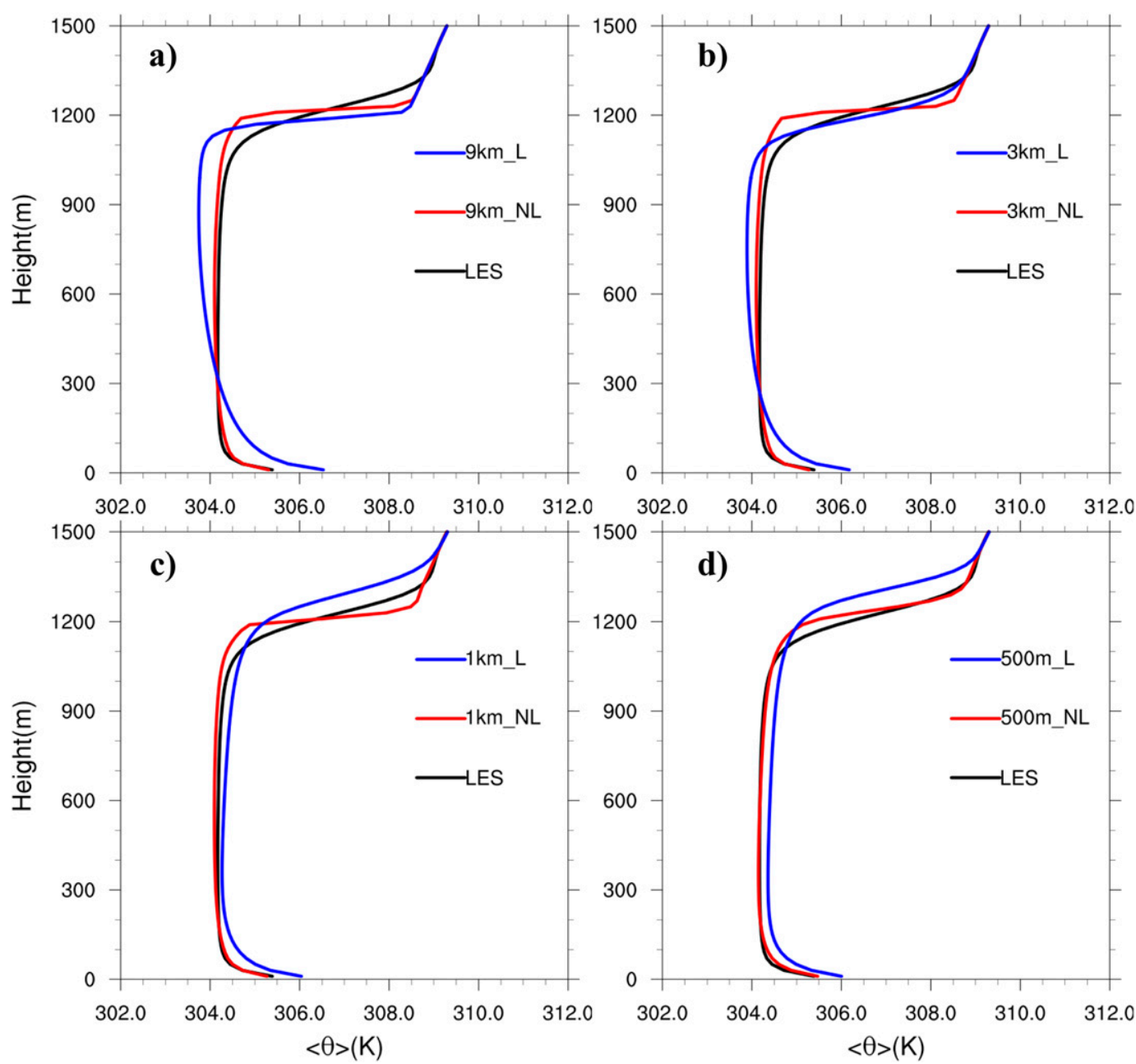

FIG. 8. Vertical profiles of potential temperature at resolutions of (a) $9 \mathrm{~km}$, (b) $3 \mathrm{~km}$, (c) $1 \mathrm{~km}$, and (d) $500 \mathrm{~m}$. The black lines are LES results, the red lines are from the new 3DTKE scheme (including the nonlocal heat flux term), and the blue lines are from the new 3DTKE scheme without nonlocal heat flux term.

deformation formula have been proposed (Lilly 1962; Xu 1988; Janjić 1990). For example, Janjić (1990) proposed a modified Smagorinsky-type parameterization for the horizontal diffusion to blend with a TKE term that is to account for the effects of horizontal diffusion caused by unresolved physical processes (e.g., turbulent entrainment and detrainment). The horizontal diffusion is strongly related to the vertical diffusion, especially for the gray zone; they need to be treated in a physically consistent way.

\section{Idealized CBL simulations}

\section{a. Experiment setup}

To evaluate the performance of the newly developed 3DTKE model, we compare it with that of the conventional 1D TKE-based PBL schemes, and with CBL LES results. Three PBL parameterizations in the WRF Model are used in this study: the Mellor-Yamada-Janjić (MYJ; Janjić 2001), the Mellor-Yamada-NakanishiNiino [MYNN; including the level-2.5 (MYNN2.5) and level-3 (MYNN3) versions; Nakanishi and Niino (2004, 2006, 2009)], and the BouLac (Bougeault and Lacarrere 1989) schemes. To test the performance of the newly developed 3DTKE scheme in the mesoscale, experiments at resolutions of $9 \mathrm{~km}, 3 \mathrm{~km}, 1 \mathrm{~km}$, and $500 \mathrm{~m}$ were conducted.

\section{b. Temperature and heat flux profiles}

Figure 4 shows the vertical profiles of horizontally averaged potential temperature at $4 \mathrm{~h}$ for the newly developed 3DTKE, MYJ, MYNN2.5, MYNN3, and BouLac experiments at various resolutions in comparison with the LES. All the experiments produce cooler 

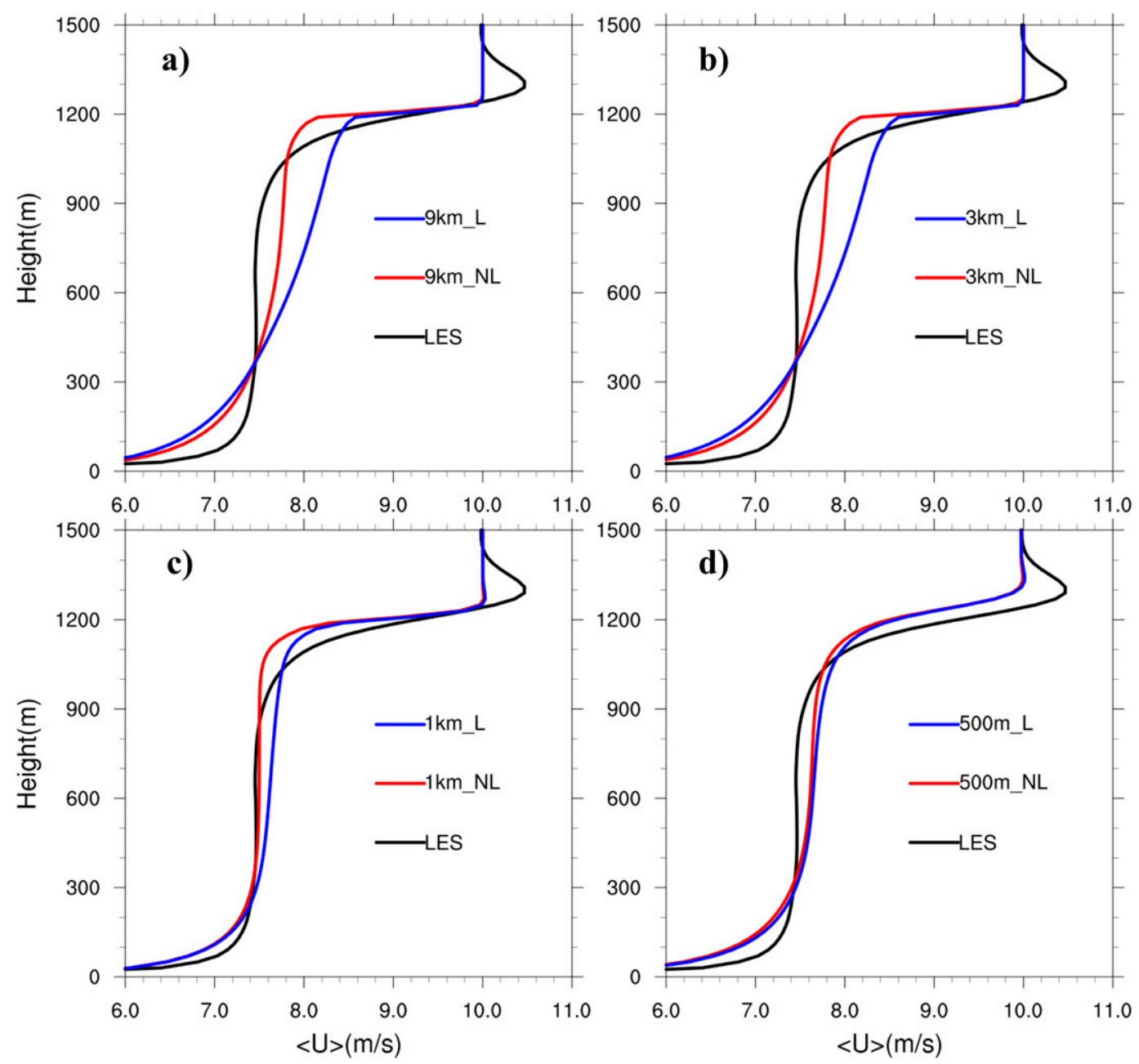

FIG. 9. As in Fig. 8, but for the mean wind.

mixed-layer temperatures than the LES profile, except for the newly developed 3DTKE scheme. For the 3DTKE experiments, the temperature profiles are almost identical to the LES profile, except in the entrainment zone. As the resolution increases, the profiles of potential temperature with the MYJ, MYNN2.5, and MYNN3 schemes become closer to the LES profile. The temperature profiles do not converge across the gray zone, except for the newly developed 3DTKE scheme and BouLac scheme, in which the horizontal grid size has little effect on the mean profiles of the potential temperature. The MYJ and MYNN2.5, the local schemes, maintain an unstable profile in order to produce a vertical heat flux through the local vertical gradient of temperature, especially for the relatively coarse resolutions ( 9 and $3 \mathrm{~km}$ ).

Figure 5 presents the vertical profiles of total vertical heat flux for the five experiments in comparison with the LES. The heat flux profiles for the newly developed
3DTKE and MYNN experiments are almost identical to the LES profiles. In the entrainment zone, all the PBL schemes underestimate the heat flux, but the newly developed 3DTKE scheme can capture the negative heat flux. Efstathiou and Beare (2015) suggested that the additional entrainment is bringing in warmer air from the free atmosphere during the course of the integration, increasing the mean temperature in the CBL. The underestimation of warm air entrainment at the top of the boundary layer might help explain why the PBL experiments produce cooler mixed-layer temperatures (Figs. 4b-e), except for the newly developed 3DTKE scheme (Fig. 4a).

The comparisons between the newly developed 3DTKE scheme and conventional 1DTKE-based PBL schemes indicate that the new 3DTKE scheme can be used in mesoscale simulations, and its performance is comparable to the conventional 1D PBL schemes. 


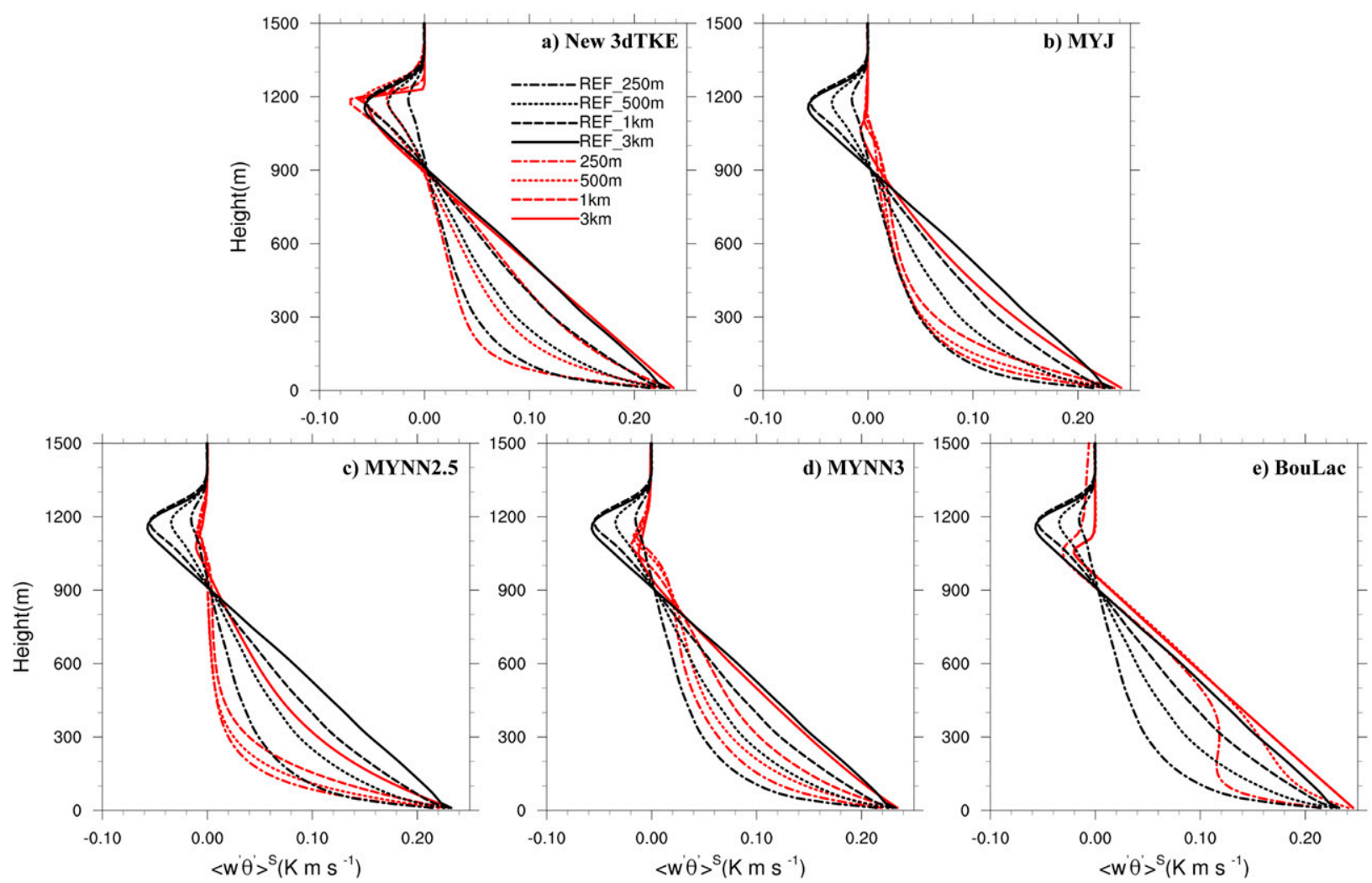

FIG. 10. Vertical profiles of horizontally averaged SGS heat flux for the (a) newly developed 3DTKE scheme, (b) MYJ, (c) MYNN2.5, (d) MYNN3, and (e) BouLac experiments at resolutions of $3 \mathrm{~km}$ (solid), $1 \mathrm{~km}$ (dashed), $500 \mathrm{~m}$ (dotted), and $250 \mathrm{~m}$ (dot-dashed). The red and black lines indicate results for experiments and coarse-grained LES, respectively.

\section{c. Wind and momentum flux profiles}

The vertical profiles of the horizontally averaged velocities $\bar{u}$ and total vertical momentum flux are shown in Figs. 6 and 7, respectively. All the PBL schemes generally underestimate the velocities in the mixed layer, except for the newly developed 3DTKE scheme. These underestimations in PBL schemes might be due to overestimations of surface drag (Fig. 7). Shin and Hong (2011) suggested that the magnitude of surface drag computed by the surface layer parameterization determines the simulated mean wind speed. Shin and Dudhia (2016) evaluated five PBL schemes and also found that the momentum profiles are underestimated in PBL schemes.

The $\bar{u}$ mean profiles for the new 3DTKE scheme are the most similar to the LES profile. The $\bar{u}$ profile is well mixed with a small gradient in the new 3DTKE scheme, while in the PBL schemes it increases roughly linearly with height to its geostrophic value. The wind profile mainly depends on the vertical gradient of momentum flux, not the magnitude of momentum flux. The results show that the newly developed 3DTKE scheme can reproduce the mean profile of velocity and momentum flux well, indicating that the new 3DTKE scheme is appropriate for mesoscale simulations.

\section{d. Sensitivities to the nonlocal flux term}

To make the original 3DTKE model suitable for mesoscale simulations, the nonlocal terms (including nonlocal heat/momentum flux) were added to the 3DTKE model. It is necessary to investigate the role of nonlocal terms in the new 3DTKE scheme.

The role of nonlocal heat flux is presented in Fig. 8, which compares the vertical profiles of potential temperature from the newly developed 3DTKE scheme with and without nonlocal heat flux at various resolutions. At relatively coarse resolutions (Figs. 8a and $8 \mathrm{~b}$ ), the role of the nonlocal heat flux is more significant, which is consistent with the above theoretical analysis. Without considering the nonlocal effect, the simulated thermal profile needs to be unstable in order to maintain the upward heat flux. The relative importance of the nonlocal effect decreases with increasing $l_{e} / \Delta$. 


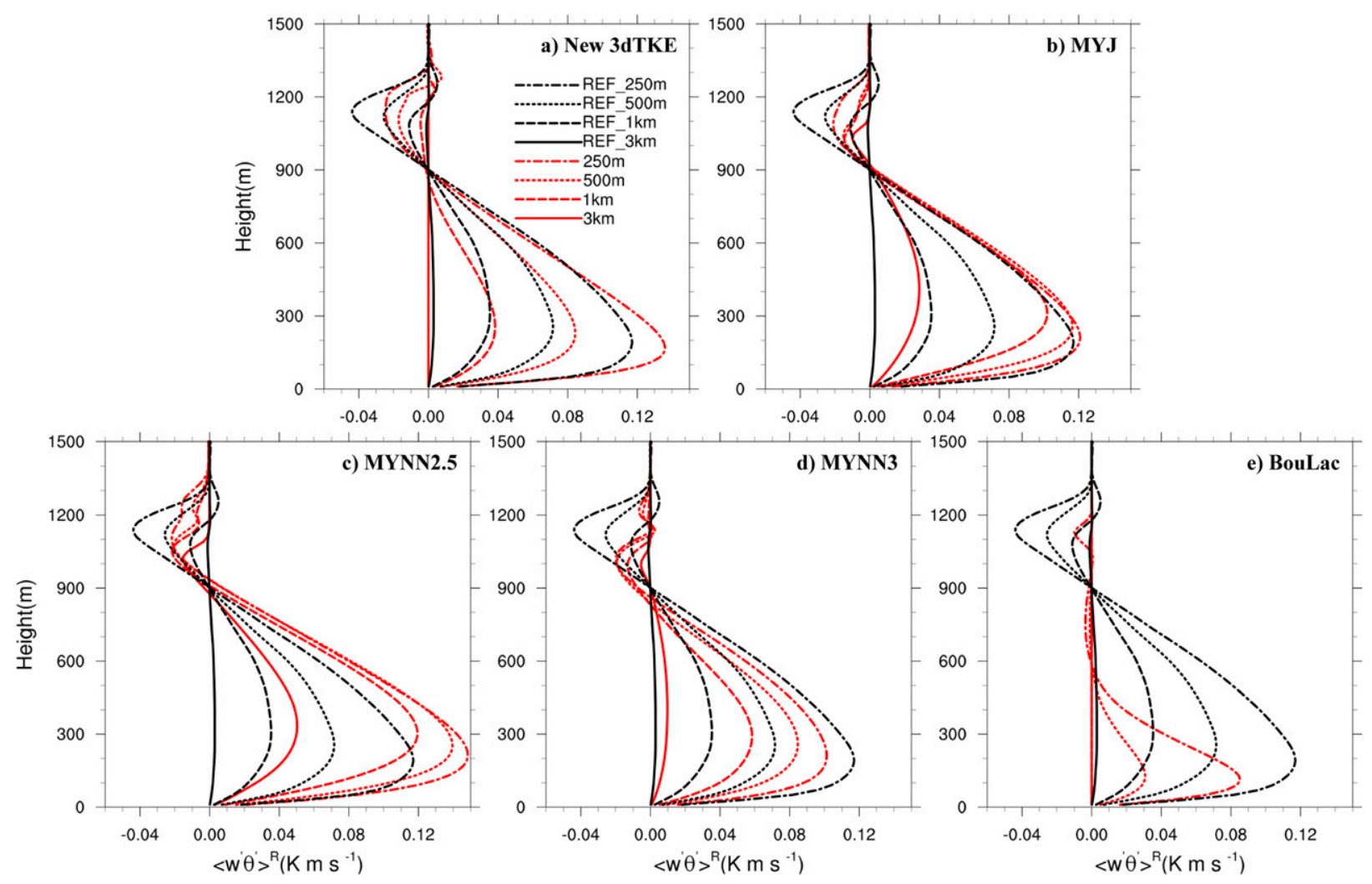

FIG. 11. As in Fig. 10, but for the resolved heat flux.

Figure 9 shows the vertical profiles of mean velocities $\bar{u}$ from the new 3DTKE model with and without nonlocal momentum flux. The neglect of the nonlocal momentum flux results in stronger shear in the mean velocity profile, particularly at coarser resolutions. As for the heat flux, the influence of the nonlocal momentum flux becomes insignificant as the resolution increases.

\section{e. SGS/resolved partitioning in the gray zone}

To evaluate the newly developed 3DTKE scheme in the gray zone, experiments at resolutions of $3 \mathrm{~km}, 1 \mathrm{~km}$, $500 \mathrm{~m}$, and $250 \mathrm{~m}$ were conducted. Figures 10 and 11 show the vertical profiles of horizontally averaged SGS and resolved heat flux for the newly developed 3DTKE scheme; MYJ, MYNN2.5, MYNN3, and BouLac experiments at each grid size; along with the coarsegrained LES profiles. Using the new 3DTKE scheme, the profiles of the SGS and resolved heat flux are similar to those from the coarse-grained data (Figs. 10a and 11a). The SGS heat flux is slightly underestimated in comparison with the coarse-grained data (Fig. 10a), while the resolved heat flux is slightly overestimated (Fig. 11a). Some PBL schemes show good performance at particular resolutions. For example, the MYJ scheme produces SGS and resolved heat fluxes very close to the coarse-grained profiles at a resolution of $250 \mathrm{~m}$ (Figs. 10b and 11b). Overall, the 1D PBL schemes fail to reproduce the transition patterns of coarse-grained data for the SGS and resolved heat flux across the gray zone.

The difference of the SGS heat flux in each 1D PBL scheme from the coarse-grained profile is compensated for by the corresponding resolved heat flux. Therefore, the underestimated SGS heat flux will lead to an overestimation of the resolved heat flux. The profiles of total heat flux remain similar at various resolutions (Fig. 5).

Figure 12 shows the vertical profiles of horizontally averaged SGS momentum flux for the five experiments at each grid size. Although the SGS momentum flux for all experiments shows some difference from the coarsegrained data, the newly developed 3DTKE scheme shows the best performance in the gray zone.

Vertical profiles of resolved TKE from the new 3DTKE scheme (Fig. 13a) are compared with those from the 1D TKE-based PBL schemes (Figs. 13b-e) against the corresponding derived TKE using the coarse-grained method (black lines). Profiles from the PBL schemes exhibit irregular shapes (Figs. 13b-e). 


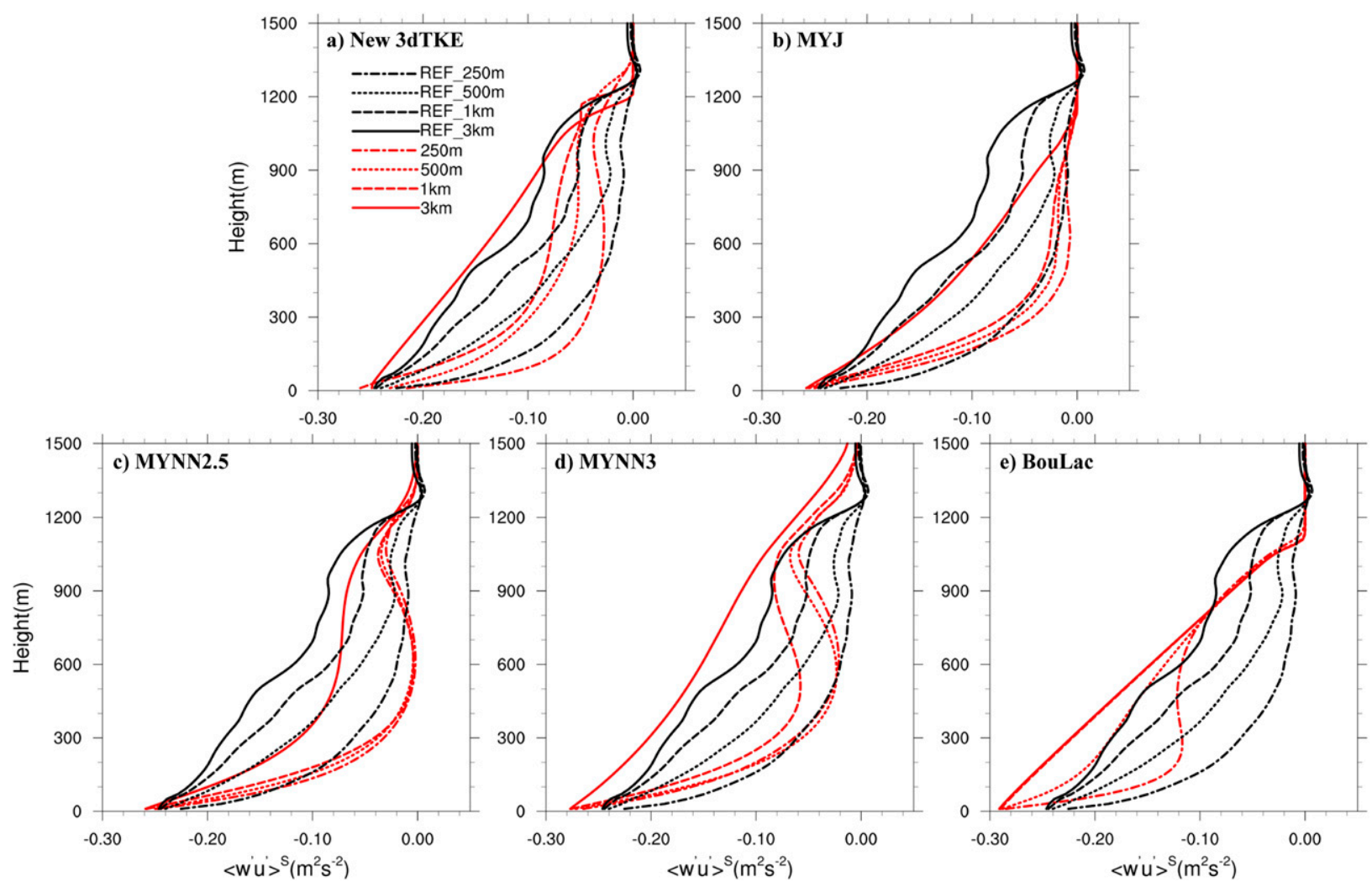

FIG. 12. As in Fig. 10, but for the SGS momentum flux.

Despite some differences, the vertical TKE profiles in the new 3DTKE scheme exhibit the same behavior as the coarse-grained data (i.e., resolved turbulence is reduced as the grid size increases) (Fig. 13a). It should be noted that in this case study of an idealized CBL, since the new 3DTKE scheme is developed based on the LES of the same idealized CBL and the same model, the results from the comparison of coarse-resolution simulations with the LES output may not really be independent. So, a more independent real-case evaluation is shown in the next section.

\section{Real-case simulations}

To examine the performance of the new 3DTKE scheme in a real-case simulation, a case with fairweather conditions is simulated using the new scheme implemented in the WRF-ARW, version 3.8.1, run in one-way nested mode. Simulations with two conventional PBL schemes were also conducted to compare with the new 3DTKE scheme. The PBL schemes used were the MYNN2.5 and YSU parameterizations. Other physical parameterizations used include a bulk microphysics scheme based on Thompson et al. (2008), the
Rapid Radiative Transfer Model with GCM applications (RRTMG) shortwave radiation scheme (Iacono et al. 2008), and the RRTM longwave parameterization scheme (Mlawer et al. 1997). No subgrid convective parameterization scheme is used on either domain. The new 3DTKE scheme, like any of the PBL schemes in the WRF Model, represents subgrid mixing through the prognostic TKE and the parameterized TKE-dependent subgrid turbulent fluxes of momentum and scalar variables. WRF does not include any parameterization of subgrid cloud formation, with grid-scale condensation being simulated by the aforementioned Thompson scheme.

The horizontal grid size (number of points) of the three domains are $3 \mathrm{~km}(793 \times 853), 1 \mathrm{~km}(805 \times 805)$, and $500 \mathrm{~m}(1001 \times 1001)$, respectively (Fig. 14). Initial conditions for the $3-\mathrm{km}$ resolution run are from the National Centers for Environmental Prediction (NCEP) Global Forecast System (GFS) analysis data on a $0.5^{\circ} \times$ $0.5^{\circ}$ grid. The lateral boundaries are from GFS forecast fields, and the time interval between forecasts is $6 \mathrm{~h}$. Initial and lateral boundary conditions for the finer-grid runs $(1 \mathrm{~km}$ and $500 \mathrm{~m})$ are obtained from coarse grid runs $(3$ and $1 \mathrm{~km})$. Each simulation was run for $24 \mathrm{~h}$, starting at 0000 UTC 29 August 2016. 


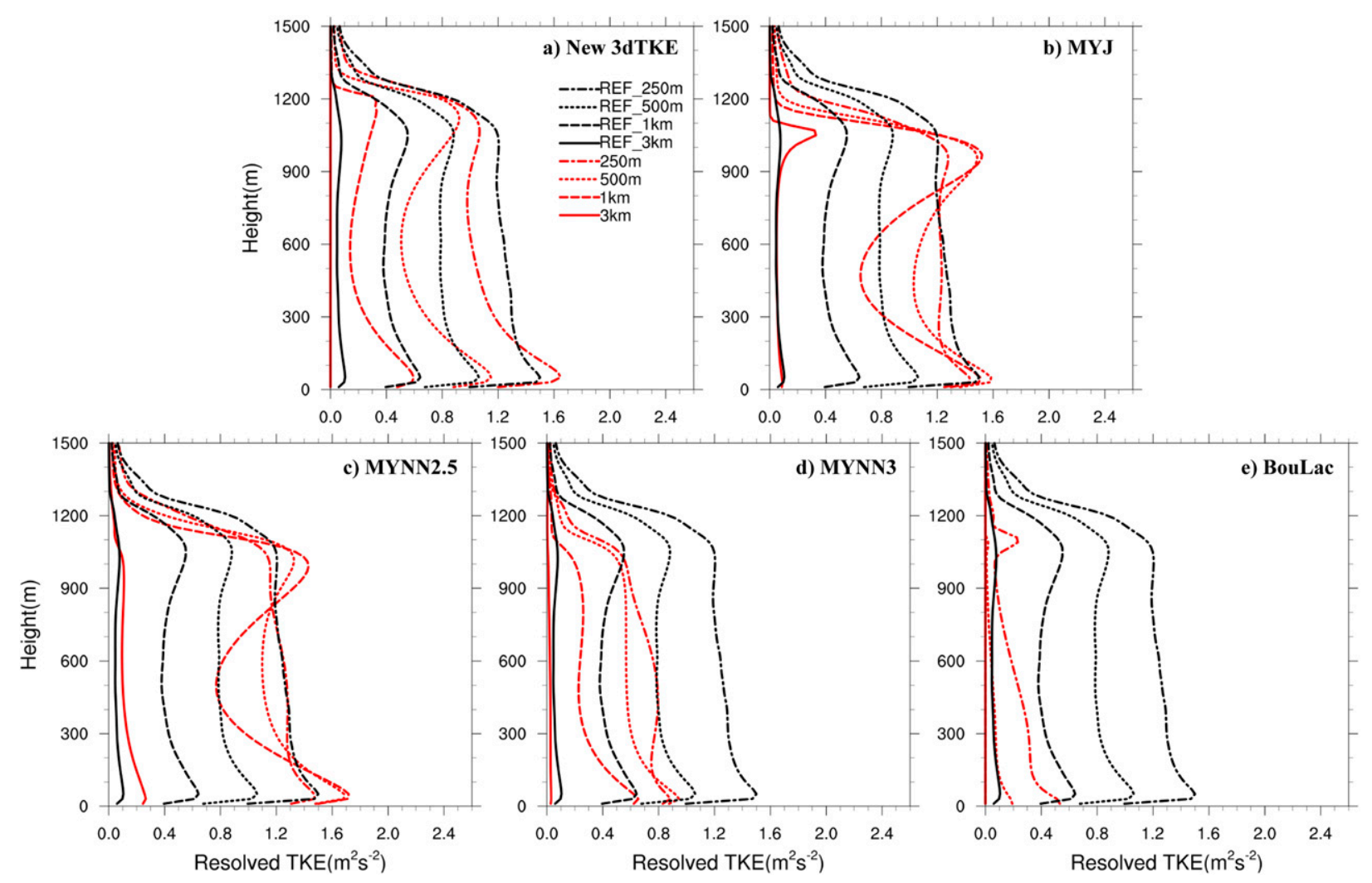

FIG. 13. As in Fig. 10, but for the resolved TKE.

Figure 15 shows snapshots of vertical velocity at about $1 \mathrm{~km}$ above ground level (AGL) at 0500 UTC for simulations with $1-\mathrm{km}$ and $500-\mathrm{m}$ resolutions, for the MYNN2.5 and YSU PBL schemes as well as the new 3DTKE scheme. The southeast-northwest convective roll-type structures are produced in all runs. In the new 3DTKE $1-\mathrm{km}$ run, the intensity of the vertical motion is stronger than in the YSU, and weaker than in the MYNN2.5 run. Shin and Hong (2015) also showed that the intensity of vertical motions, both updrafts and downdrafts, is strengthened using their new parameterization compared to the YSU scheme. The results indicate that reasonable convective roll structures are produced by the new 3DTKE scheme, whose performance is comparable to the conventional PBL schemes. Similarly, LeMone et al. (2010) simulated convective roll structures using 1-km grid spacing and $1 \mathrm{D}$ PBL schemes that were in good agreement with satellite images.

At a resolution of $500 \mathrm{~m}$, the roll structures seen in the 1-km run almost disappear in the MYNN2.5 run (Fig. 15d), and convective cells become dominant. Using the new 3DTKE scheme, the pattern of convection appears more irregular than in the $1-\mathrm{km}$ runs, but the linear roll structure is somewhat maintained (Fig. 15f). The YSU run shows similar behavior to the new 3DTKE run, but with weaker intensity (Fig. 15e). A 250-m resolution visible Moderate Resolution Imaging Spectroradiometer (MODIS)-Terra satellite image at 0240 UTC 29 August is shown in Fig. 16. The simulated horizontal roll-like structures (Fig. 15) are similar in scale and appearance to the satellite cloud field. The cloud streets depicted in the satellite image have linear roll structures with the north-northwest-south-southeast orientation similar to the model results. Likewise, LeMone et al. (2010) found that a finer grid size can lead to more irregular convection, with the roll structure becoming more cellular with smaller grid size. Using the MYJ scheme, Trier et al. (2004) also found the type of convection changed from linear to more cellular when the grid size decreased. The vertical velocity is shown in Fig. 15 instead of the simulated cloud field because there is a low bias in the cloud water content in the model output. The lack of boundary layer clouds in the realcase simulations is due to the overall dry biases in both the model initial state and simulation (not shown).

Figure 17 shows vertical profiles of simulated potential temperature for runs with $1-\mathrm{km}$ and 500-m resolutions 


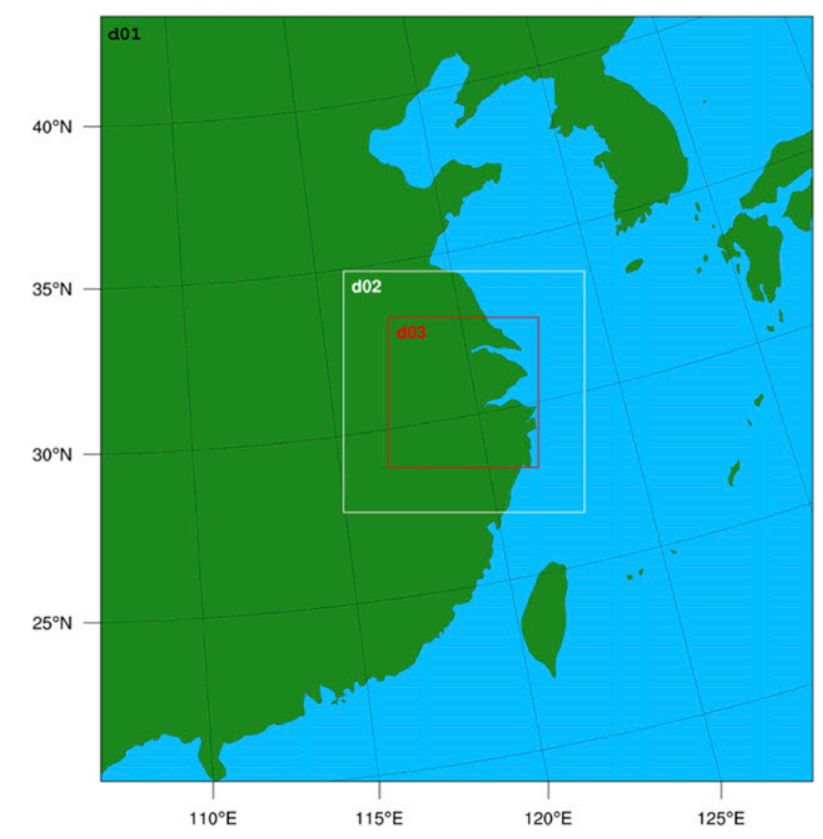

FIG. 14. Model domains for real-case simulations. Outermost domain (d01) resolution is $3 \mathrm{~km}$, intermediate domain $(\mathrm{d} 02)$ is $1 \mathrm{~km}$, and innermost domain (d03) is $500 \mathrm{~m}$.

at 0500 UTC (1400 LST) 29 August at Baoshan, Shanghai, China $\left(31.40^{\circ} \mathrm{N}, 121.45^{\circ} \mathrm{E}\right)$, with corresponding radiosonde measurements. The observed potential temperature profile is near neutral. In the $1-\mathrm{km}$ runs, the new 3DTKE and YSU runs produce well-mixed boundary layers, while the thermal profile is quite unstable in the MYNN2.5 run because of its local mixing approach. In the 500-m runs, the profile in the new 3DTKE run is weakly stable and close to the observed, although it is slightly cooler than the YSU run. The MYNN2.5 underestimates the temperature by approximately $2 \mathrm{~K}$ in the mixed layer.

Figure 18 shows the time series of the simulated 2-m temperature compared to observations. The values are averaged over 10 stations within the d03 domain. In the $1-\mathrm{km}$ runs, the time series of $2-\mathrm{m}$ temperature in all the runs are similar but all indicate a cold bias. In the 500-m runs, the result in the new 3DTKE run is nearly identical to that in the YSU run. The MYNN2.5 run shows an even greater cold bias (about $3 \mathrm{~K}$ ) compared to the observed temperatures. Through the simulation intercomparison and comparison with observations, the new 3DTKE scheme shows the feasibility of replacing the conventional PBL schemes.

The results from this real-case experiment show that for the new 3DTKE scheme, the temperature profiles and time series remain nearly identical, regardless of the model's resolution. It reduces the effect of the parameterization as the model's resolution increases. The YSU scheme, which was originally designed for mesoscale modeling, also shows the insensitivity of temperature to resolution, but appears to prevent the dynamics from resolving any additional features. The MYNN scheme tends to allow more resolved features at the gray zone, but the results seem unreasonable.

\section{Conclusions and discussion}

As the resolution of numerical weather prediction models steadily increases, such that grid size becomes comparable to the typical size of the largest energycontaining eddies, the development of a more general treatment of horizontal and vertical subgrid turbulent mixing is required to overcome the energetic inconsistency of the conventional parameterization approach. To this end, a coherent, generalized parameterization scheme based on the full 3D TKE prognostic equation has been developed in WRF. In this study, the original 3DTKE model that is commonly used as the LES SGS model is extended to the mesoscale limit. An algorithm for transitioning between LES and mesoscale limits was presented, along with specific closure assumptions for the determinations of nonlocal heat and momentum fluxes, the master mixing length, and the horizontal diffusion. The results from idealized CBL and real-case simulations show that the new 3DTKE scheme can be used in mesoscale simulations, and its performance is comparable to the conventional 1D PBL schemes. For simulations with resolutions ranging from $3 \mathrm{~km}$ to $250 \mathrm{~m}$, the newly developed 3DTKE scheme led to improved representation of CBL heat and momentum fluxes, as well as potential temperature profiles. The scheme is appropriate for the LES and mesoscale limits, and for the range of scales in between.

The 3D subgrid mixing parameterization is needed, especially for the region where the assumption of horizontal homogeneity is violated (e.g., complex terrain or sea breeze). Recently, Muñoz-Esparza et al. (2016) discussed the limitations of 1D PBL schemes in reproducing mountain-wave flow, and suggested the necessity to develop 3D PBL schemes. The newly developed 3DTKE scheme in this study provides the possibility to consider the all the components of the Reynolds stress tensor in a coherent, more general framework. The development makes it feasible to replace conventional 1D PBL schemes with the scaleadaptive 3D TKE-based scheme.

In this study, we introduced a pragmatic blending approach for the transition of horizontal diffusion across 


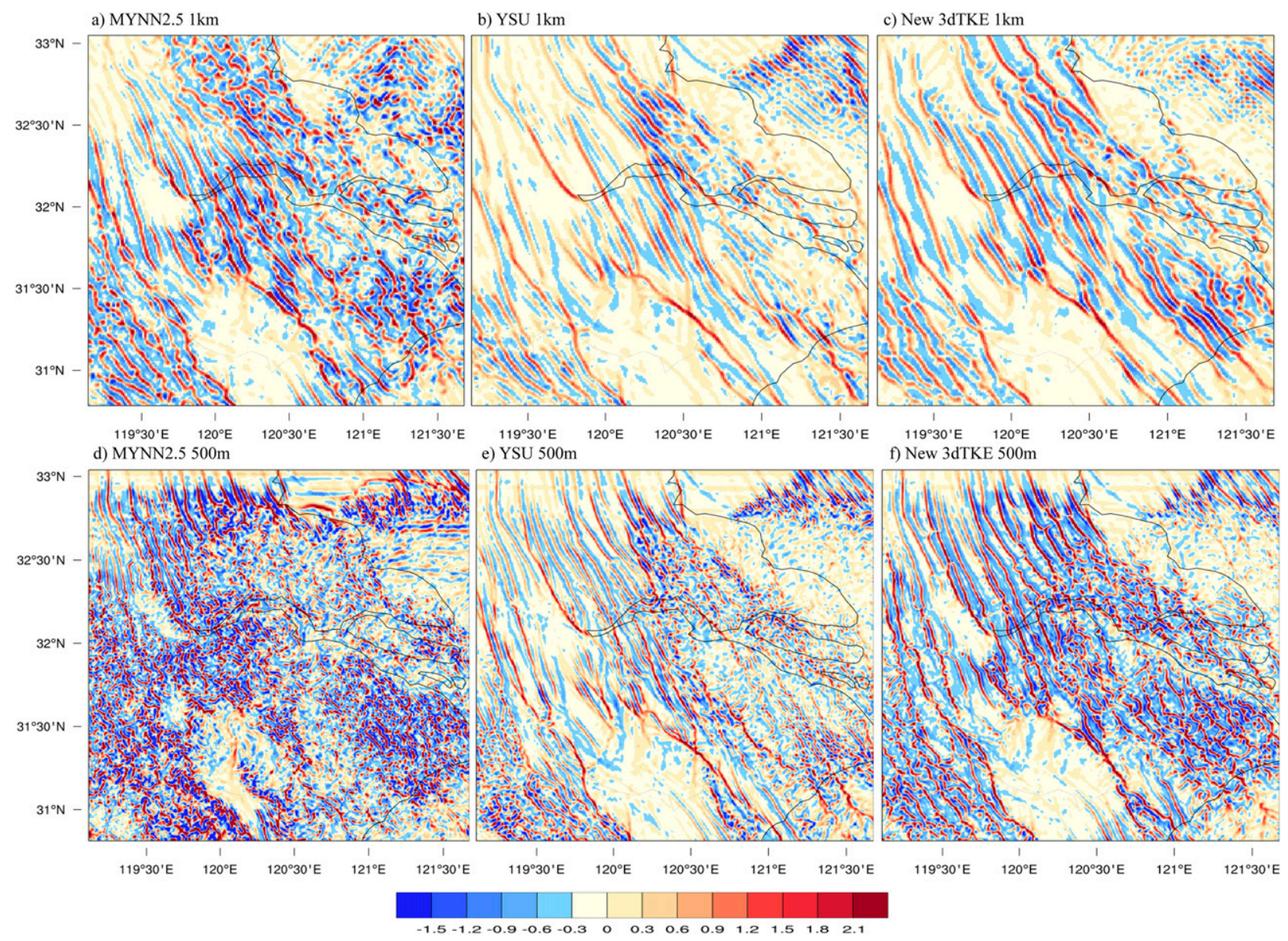

FIG. 15. Horizontal distributions of vertical velocity at $z=1 \mathrm{~km}$ over the d03 subdomain at 0500 UTC 29 Aug 2016, for the 1-km resolution runs of (a) MYNN2.5, (b) YSU, and (c) new 3DTKE, and for the 500-m resolution runs of (d) MYNN2.5, (e) YSU, and (f) new 3DTKE.

the gray zone, which blends the Smagorinsky-Lilly closure based on the deformation for mesoscale simulation, with a TKE-based subgrid mixing scheme for LES. However, in the gray zone, there are no proper horizontal diffusion parameterizations in numerical models yet. It is important to note that Ito et al. (2014) derived the horizontal eddy diffusivity in a CBL and presented vertical profiles of horizontal eddy diffusivity and length scale, but the eddy diffusivity is only applicable to scalar-field gradients on horizontal scales larger than the convection cells. That is, there is no scale transition for the derived horizontal eddy diffusivity to be applicable to the resolutions close to the LES limit. Some explicit horizontal diffusion processes are implemented for mostly numerical purposes (e.g., to remove small-scale noise with wavelengths of 2-4 times the grid intervals; Xue 2000; Knievel et al. 2007; Langhans et al. 2012). Others are meant to mimic SGS turbulence processes that are unresolved (Smagorinsky
1963). In practice, many horizontal diffusion processes are used at once in the numerical model, which makes it more difficult to distinguish their individual effects.

Recently, multiple studies have addressed the importance of horizontal diffusion in more realistic cases, and attempted to evaluate and quantify the horizontal diffusion based on more physical processes (Bryan and Rotunno 2009; Rotunno and Bryan 2012; Bryan 2012; Machado and Chaboureau 2015). Honnert (2016) calculated the horizontal mixing length from LES of neutral and convective cases in the gray zone resolutions. The parameterization of horizontal diffusion is still one of the most uncertain aspects in NWP models; there is no theory for how to quantify the intensity of horizontal diffusion, and few observations are available to constrain the adjustable settings (e.g., horizontal mixing length $l_{h}$ ). Hanley et al. (2015) investigated the sensitivities of the storm morphology and statistical properties to the horizontal diffusion, and evaluated it against 


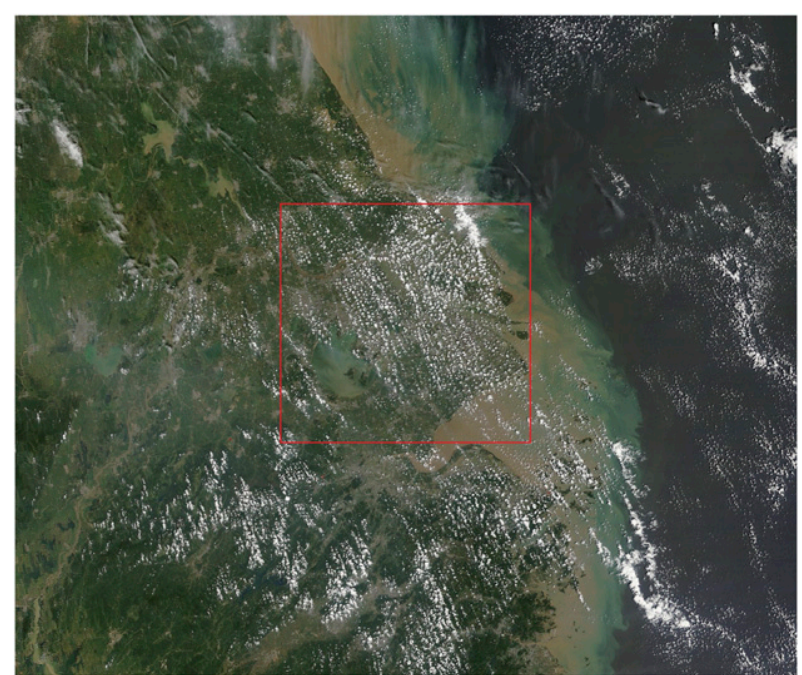

FIG. 16. The 250-m resolution visible MODIS-Terra image at 0240 UTC 29 Aug 2016. The image is taken from online (http:// lance.nasa.gov/imagery/rapid-response), and the red box indicates the domain shown in Fig. 15.

the Met Office rainfall radar network. Evaluating and developing the horizontal diffusion parameterization using observations may be the future direction.

Because we sought to identify the key issues in simulating the boundary layer and utilize them to design the new 3DTKE scheme aiming at scale adaptivity, the analysis of LES data is limited to the dry CBL. Even though the newly developed 3DTKE scheme improved the gray zone simulation of the idealized CBL, the complexity of the real world is such that the performance of the new scheme turned out to be neutral in the real-case evaluation, indicating that there are remaining issues in the new scheme that need to be addressed in the future. For example, the analysis of a cloud-topped boundary layer LES is also needed to evaluate the algorithm used in this study. In addition, the background diffusion and the free atmospheric mixing above the PBL are also important in real-weather forecasting. All these will be addressed in future work.

Acknowledgments. We thank two anonymous reviewers for their careful review of our manuscript and their many insightful comments and suggestions. This work is supported by the National Key Research and Development Program of China (2016YFE0109700) and the National Science Foundation of China (Grants 41505087 and 41575101). Xu Zhang is grateful to the Physical Sciences Division of NOAA's Earth System Research Laboratory in Boulder, Colorado, for hosting his visit between December 2015 and July 2016, which was critical to the inception of the manuscript. Coauthors Jian-Wen Bao and Evelyn D. Grell thank Melinda Marquis, of the Global Systems Division in NOAA's Earth System Research Laboratory, for her support of their work on the subject of the manuscript through the Atmospheric Science for Renewable Energy Development Program. In addition, we acknowledge the use of Rapid Response imagery from the Land,

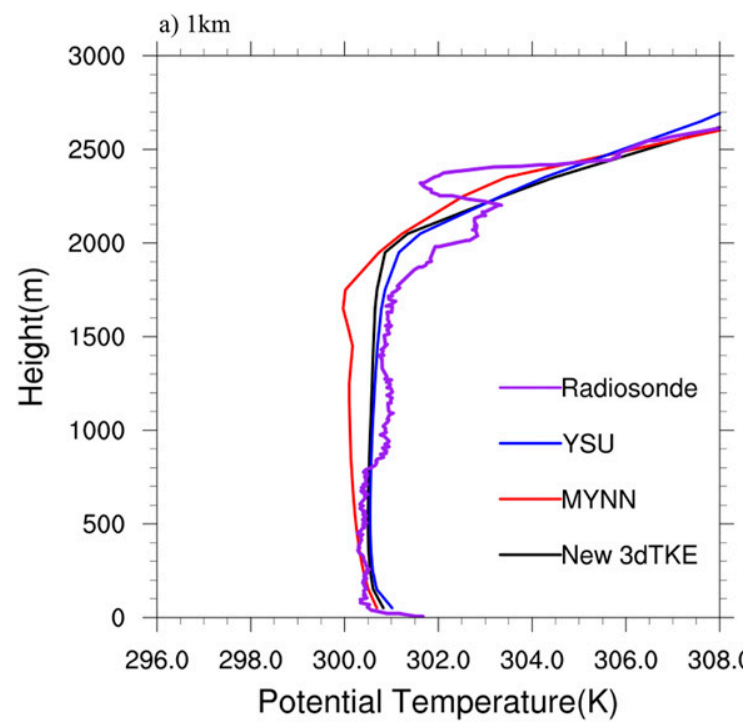

b) $500 \mathrm{~m}$

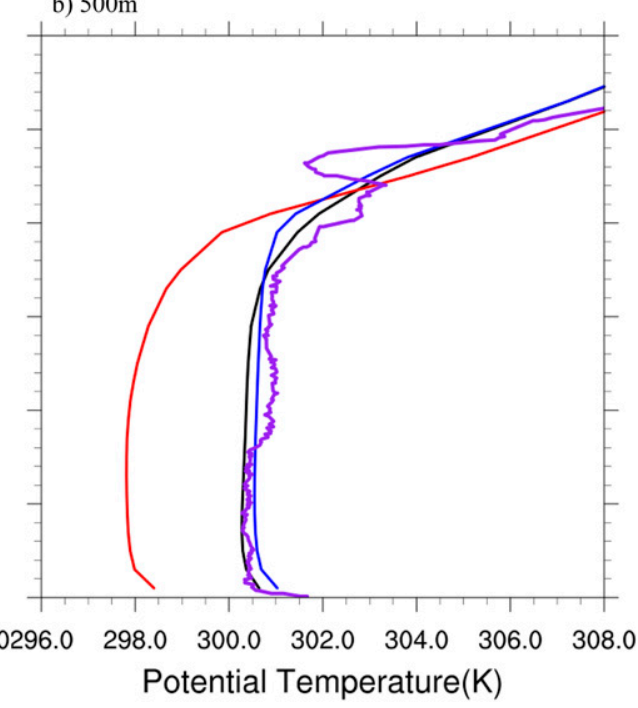

FIG. 17. Vertical profiles of simulated potential temperature at 0500 UTC 29 Aug 2016 for the Baoshan station $\left(31.40^{\circ} \mathrm{N}, 121.45^{\circ} \mathrm{E}\right)$ along with the corresponding radiosonde (purple). The simulated results are from the YSU (blue), MYNN2.5 (red), and new 3DTKE (black) experiments with the resolutions of (a) $1 \mathrm{~km}$ and (b) $500 \mathrm{~m}$. 

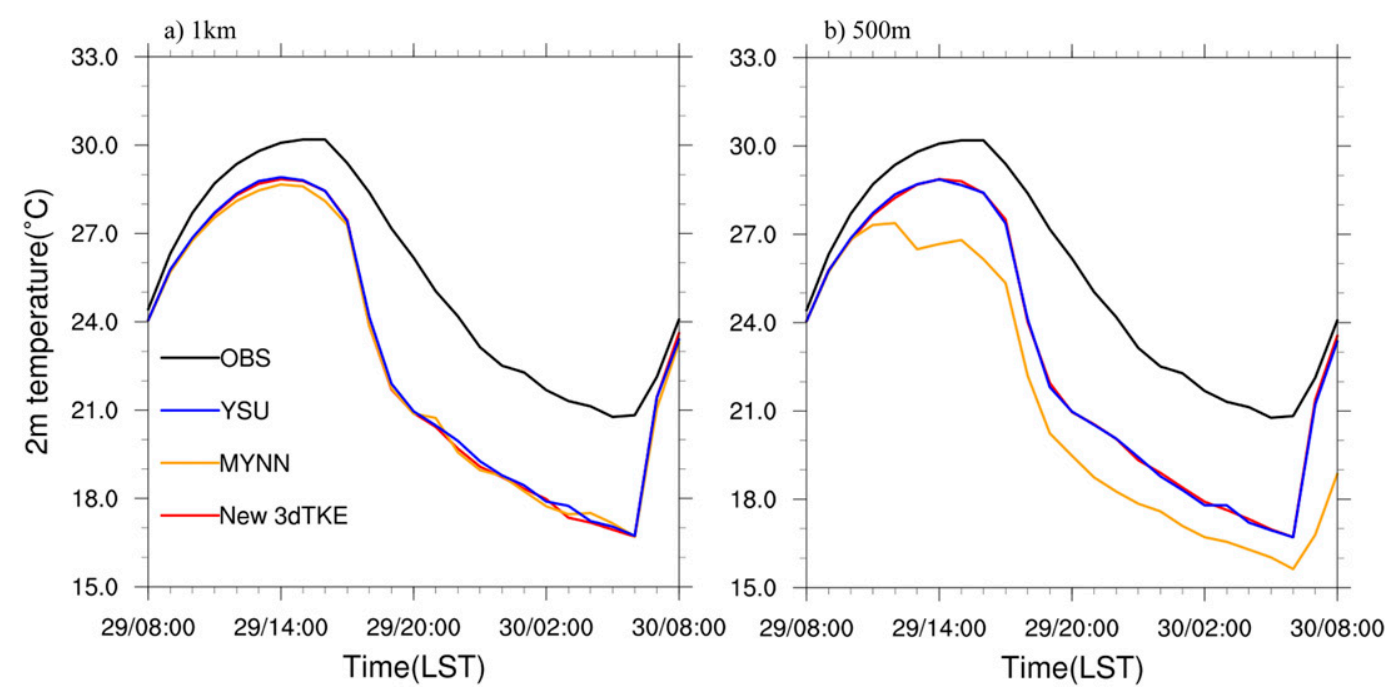

FIG. 18. Time series of simulated 2-m temperature $\left({ }^{\circ} \mathrm{C}\right)$ along with the corresponding observation (black). The simulated results are from the YSU (blue), MYNN2.5 (yellow), and new 3DTKE (red) runs with the resolutions of (a) $1 \mathrm{~km}$ and (b) $500 \mathrm{~m}$. The result is an average over 10 stations in eastern China within the d03 domain.

Atmosphere Near real-time Capability for EOS (LANCE) system operated by the NASA/GSFC/Earth Science Data and Information System (ESDIS) with funding provided by NASA/HQ.

\section{APPENDIX}

\section{Partition Functions for Heat Flux and Turbulent Kinetic Energy}

The partition functions are obtained by fitting to the LES data. The functions are nearly identical to those of Shin and Hong (2015), except a few constants are tuned to fit our LES data. By fitting the LES data, the partition functions of the SGS nonlocal and local flux in the mixed layer (i.e., $0.2 \leq z / z_{i} \leq 0.6$ ) are given as

$P_{\mathrm{NL}}\left(\Delta / z_{i}\right)=0.243 \frac{\left(\Delta / z_{i}\right)^{2}+0.936\left(\Delta / z_{i}\right)^{7 / 8}-1.11}{\left(\Delta / z_{i}\right)^{2}+0.312\left(\Delta / z_{i}\right)^{7 / 8}+0.329}+0.757$

and

$P_{L}\left(\Delta / z_{i}\right)=0.280 \frac{\left(\Delta / z_{i}\right)^{2}+0.870\left(\Delta / z_{i}\right)^{1 / 2}-0.913}{\left(\Delta / z_{i}\right)^{2}+0.153\left(\Delta / z_{i}\right)^{1 / 2}+0.278}+0.720$

The resulting curves are presented in Fig. 3.

The partition function of TKE is identical to that of Honnert et al. (2011), which is given as
$P_{\mathrm{TKE}}\left(\Delta / z_{i}\right)=\frac{\left(\Delta / z_{i}\right)^{2}+0.07\left(\Delta / z_{i}\right)^{2 / 3}}{\left(\Delta / z_{i}\right)^{2}+0.142\left(\Delta / z_{i}\right)^{2 / 3}+0.071}$.

For simplification, the height dependency of the partition functions is ignored, only partition functions in the mixed layer are used in this study.

\section{REFERENCES}

Antonelli, M., and R. Rotunno, 2007: Large-eddy simulation of the onset of the sea breeze. J. Atmos. Sci., 64, 4445-4457, https:// doi.org/10.1175/2007JAS2261.1.

Bougeault, P., and P. Lacarrere, 1989: Parameterization of orography-induced turbulence in a mesobeta-scale model. Mon. Wea. Rev., 117, 1872-1890, https://doi.org/10.1175/ 1520-0493(1989)117<1872:POOITI>2.0.CO;2.

Boutle, I. A., J. E. J. Eyre, and A. P. Lock, 2014: Seamless stratocumulus simulation across the turbulent gray zone. Mon. Wea. Rev., 142, 1655-1668, https://doi.org/10.1175/ MWR-D-13-00229.1.

Brown, A. R., and A. L. M. Grant, 1997: Non-local mixing of momentum in the convective boundary layer. Bound.-Layer Meteor., 84, 1-22, https://doi.org/10.1023/A:1000388830859.

Bryan, G. H., 2012: Effects of surface exchange coefficients and turbulence length scales on the intensity and structure of numerically simulated hurricanes. Mon. Wea. Rev., 140, 11251143, https://doi.org/10.1175/MWR-D-11-00231.1.

— , and R. Rotunno, 2009: The maximum intensity of tropical cyclones in axisymmetric numerical model simulations. Mon. Wea. Rev., 137, 1770-1789, https://doi.org/10.1175/ 2008MWR2709.1.

—, J. C. Wyngaard, and J. M. Fritsch, 2003: Resolution requirements for the simulation of deep moist convection. Mon. Wea. Rev., 131, 2394-2416, https://doi.org/10.1175/ 1520-0493(2003)131<2394:RRFTSO > 2.0.CO;2. 
Catalano, F., and C.-H. Moeng, 2010: Large-eddy simulation of the daytime boundary layer in an idealized valley using the Weather Research and Forecasting numerical model. Bound.-Layer Meteor., 137, 49-75, https://doi.org/10.1007/ s10546-010-9518-8.

Conzemius, R. J., and E. Fedorovich, 2006: Dynamics of sheared convective boundary layer entrainment. Part II: Evaluation of bulk model predictions of entrainment flux. J. Atmos. Sci., 63, 1179-1199, https://doi.org/10.1175/JAS3696.1.

Couvreux, F., F. Hourdin, and C. Rio, 2010: Resolved versus parametrized boundary-layer plumes. Part I: A parametrization oriented conditional sampling in large-eddy simulations. Bound.-Layer Meteor., 134, 441-458, https://doi.org/10.1007/ s10546-009-9456-5.

Deardorff, J. W., 1972: Theoretical expression for the countergradient vertical heat flux. J. Geophys. Res., 77, 5900-5904, https://doi.org/10.1029/JC077i030p05900.

- 1980: Stratocumulus-capped mixed layers derived from a three-dimensional model. Bound.-Layer Meteor., 18, 495-527, https://doi.org/10.1007/BF00119502.

- 1985: Sub-grid-scale turbulence modeling. Advances in Geophysics, Vol. 28, Academic Press, 337-343, https://doi.org/ 10.1016/S0065-2687(08)60193-4.

_ the entrainment zone of a convective mixed layer. J. Fluid Mech., 100, 41-64, https://doi.org/10.1017/S0022112080001000.

Efstathiou, G. A., and R. J. Beare, 2015: Quantifying and improving sub-grid diffusion in the boundary-layer grey zone. Quart. J. Roy. Meteor. Soc., 141, 3006-3017, https://doi.org/ 10.1002/qj.2585.

Greenhut, G. K., and S. J. S. Khalsa, 1982: Updraft and downdraft events in the atmospheric boundary layer over the equatorial Pacific Ocean. J. Atmos. Sci., 39, 1803-1818, https://doi.org/ 10.1175/1520-0469(1982)039<1803:UADEIT>2.0.CO;2.

Hanley, K. E., R. S. Plant, T. H. M. Stein, R. J. Hogan, J. C. Nicol, H. W. Lean, C. Halliwell, and P. A. Clark, 2015: Mixing-length controls on high-resolution simulations of convective storms. Quart. J. Roy. Meteor. Soc., 141, 272-284, https://doi.org/ 10.1002/qj.2356.

Hellsten, A., and S. Zilitinkevich, 2013: Role of convective structures and background turbulence in the dry convective boundary layer. Bound.-Layer Meteor., 149, 323-353, https:// doi.org/10.1007/s10546-013-9854-6.

Holtslag, A. A. M., and C.-H. Moeng, 1991: Eddy diffusivity and counter gradient transport in the convective atmospheric boundary layer. J. Atmos. Sci., 48, 1690-1698, https://doi.org/ 10.1175/1520-0469(1991)048<1690:EDACTI > 2.0.CO;2.

Hong, S.-Y., Y. Noh, and J. Dudhia, 2006: A new vertical diffusion package with an explicit treatment of entrainment processes. Mon. Wea. Rev., 134, 2318-2341, https://doi.org/10.1175/ MWR3199.1.

Honnert, R., 2016: Representation of the grey zone of turbulence in the atmospheric boundary layer. Adv. Sci. Res., 13, 63-67, https://doi.org/10.5194/asr-13-63-2016.

_- V. Masson, and F. Couvreux, 2011: A diagnostic for evaluating the representation of turbulence in atmospheric models at the kilometric scale. J. Atmos. Sci., 68, 3112-3131, https:// doi.org/10.1175/JAS-D-11-061.1.

Iacono, M. J., J. S. Delamere, E. J. Mlawer, M. W. Shephard, S. A. Clough, and W. D. Collins, 2008: Radiative forcing by longlived greenhouse gases: Calculations with the AER radiative transfer models. J. Geophys. Res., 113, D13103, https://doi.org/ 10.1029/2008JD009944.
Ito, J., H. Niino, and M. Nakanishi, 2014: Horizontal turbulent diffusion in a convective mixed layer. J. Fluid Mech., 758, 553 564, https://doi.org/10.1017/jfm.2014.545.

- $,-\ldots, \ldots$, and C.-H. Moeng, 2015: An extension of Mellor-Yamada model to the terra incognita zone for dry convective mixed layers in the free convection regime. Bound.-Layer Meteor., 157, 23-43, https://doi.org/10.1007/ s10546-015-0045-5.

Jablonowski, C., and D. Williamson, 2011: The pros and cons of diffusion, filters and fixers in atmospheric general circulation models. Numerical Techniques for Global Atmospheric Models, P. H. Lauritzen et al., Eds., Lecture Notes in Computational Science and Engineering, Vol. 80, Springer, 381493.

Janjić, Z. I., 1990: The step-mountain coordinate: Physical package. Mon. Wea. Rev., 118, 1429-1443, https://doi.org/10.1175/ 1520-0493(1990)118<1429:TSMCPP > 2.0.CO;2.

_ 2001: Nonsingular implementation of the Mellor-Yamada level 2.5 scheme in the NCEP Meso model. NOAA/NWS/ NCEP Office Note 437, 61 pp.

Klemp, J. B., and R. B. Wilhelmson, 1978: The simulation of three dimensional convective storm dynamics. J. Atmos. Sci., 35, 1070-1096, https://doi.org/10.1175/1520-0469(1978) 035<1070:TSOTDC $>2.0$. CO 2 .

Knievel, J. C., G. H. Bryan, and J. P. Hacker, 2007: Explicit numerical diffusion in the WRF model. Mon. Wea. Rev., 135, 3808-3824, https://doi.org/10.1175/2007MWR2100.1.

Kurowski, M., and J. Teixeira, 2018: A scale-adaptive turbulent kinetic energy closure for the dry convective boundary layer. J. Atmos. Sci., 75, 675-689, https://doi.org/10.1175/ JAS-D-16-0296.1.

Langhans, W., J. Schmidli, and C. Schär, 2012: Mesoscale impacts of explicit numerical diffusion in a convection-permitting model. Mon. Wea. Rev., 140, 226-244, https://doi.org/10.1175/ 2011MWR3650.1.

LeMone, M. A., F. Chen, M. Tewari, J. Dudhia, B. Geerts, Q. Miao, R. L. Coulter, and R. L. Grossman, 2010: Simulating the IHOP_2002 fair-weather CBL with the WRF-ARW-Noah modeling system. Part II: Structures from a few kilometers to100 km across. Mon. Wea. Rev., 138, 745-764, https://doi.org/ 10.1175/2009MWR3004.1.

Lenschow, D. H., 1974: Model of the height variation of the turbulent kinetic energy budget in the unstable planetary boundary layer. J. Atmos. Sci., 31, 465-474, https://doi.org/ 10.1175/1520-0469(1974)031<0465:MOTHVO > 2.0.CO;2.

— vective boundary layer. Bound.-Layer Meteor., 19, 509-532, https://doi.org/10.1007/BF00122351.

Lilly, D. K., 1962: On the numerical simulation of buoyant convection. Tellus, 14, 148-172, https://doi.org/10.3402/ tellusa.v14i2.9537.

_ 1967: The representation of small-scale turbulence in numerical simulation experiments. Proc. IBM Scientific Computing Symp. on Environmental Sciences, Yorktown Heights, NY, Thomas J. Watson Research Center, IBM, 195-210.

Machado, L. A., and J.-P. Chaboureau, 2015: Effect of turbulence parameterization on assessment of cloud organization. Mon. Wea. Rev., 143, 3246-3262, https://doi.org/ 10.1175/MWR-D-14-00393.1.

Mellor, G. L., 1973: Analytic prediction of the properties of stratified planetary surface layers. J. Atmos. Sci., 30, 10611069, https://doi.org/10.1175/1520-0469(1973)030<1061: APOTPO $>2.0 . \mathrm{CO} ; 2$. 
— , and T. Yamada, 1974: A hierarchy of turbulence closure models for planetary boundary layers. J. Atmos. Sci., 31, 1791-1806, https://doi.org/10.1175/1520-0469(1974)031<1791: AHOTCM $>2.0 . \mathrm{CO} ; 2$.

- and - 1982: Development of a turbulence closure model for geophysical fluid problems. Rev. Geophys. Space Phys., 20, 851-875, https://doi.org/10.1029/RG020i004p00851.

Mlawer, E. J., S. J. Taubman, P. D. Brown, and M. J. Iacono, 1997: Radiative transfer for inhomogeneous atmospheres: RRTM, a validated correlated-k model for the longwave. J. Geophys. Res., 102, 16 663-16682, https://doi.org/10.1029/97JD00237.

Moeng, C.-H., and P. P. Sullivan, 1994: A comparison of shearand buoyancy-driven planetary boundary layer flows. J. Atmos. Sci., 51, 999-1022, https://doi.org/10.1175/ 1520-0469(1994)051<0999:ACOSAB > 2.0.CO;2.

_- J. Dudhia, J. Klemp, and P. Sullivan, 2007: Examining twoway grid nesting for large eddy simulation of PBL using the WRF model. Mon. Wea. Rev., 135, 2295-2311, https://doi.org/ 10.1175/MWR3406.1.

Muñoz-Esparza, D., J. Sauer, R. Linn, and B. Kosović, 2016: Limitations of one-dimensional mesoscale PBL parameterizations in reproducing mountain-wave flows. J. Atmos. Sci., 73, 2603-2614, https://doi.org/10.1175/JAS-D-15-0304.1.

Nakanishi, M., and H. Niino, 2004: An improved Mellor-Yamada Level-3 model with condensation physics: Its design and verification. Bound.-Layer Meteor., 112, 1-31, https://doi.org/ 10.1023/B:BOUN.0000020164.04146.98.

_- and —, 2006: An improved Mellor-Yamada Level-3 model: Its numerical stability and application to a regional prediction of advection fog. Bound.-Layer Meteor., 119, 397 407, https://doi.org/10.1007/s10546-005-9030-8.

— , and 2009: Development of an improved turbulence closure model for the atmospheric boundary layer. J. Meteor. Soc. Japan, 87, 895-912, https://doi.org/10.2151/jmsj.87.895.

Noh, Y., W.-G. Cheon, S.-Y. Hong, and S. Raasch, 2003: Improvement of the K-profile model for the planetary boundary layer based on large eddy simulation data. Bound.-Layer Meteor., 107, 401-427, https://doi.org/10.1023/A:1022146015946.

Parodi, A., and S. Tanelli, 2010: Influence of turbulence parameterizations on high-resolution numerical modeling of tropical convection observed during the TC4 field campaign. J. Geophys. Res., 115, D00J14, https://doi.org/ 10.1029/2009JD013302.

Rotunno, R., and G. H. Bryan, 2012: Effects of parameterized diffusion on simulated hurricanes. J. Atmos. Sci., 69, 2284 2299, https://doi.org/10.1175/JAS-D-11-0204.1.

Schmidt, H., and U. Schumann, 1989: Coherent structure of the convective planetary boundary layer. J. Fluid Mech., 200, 511562, https://doi.org/10.1017/S0022112089000753.

Shin, H. H., and S.-Y. Hong, 2011: Intercomparison of planetary boundary-layer parametrizations in the WRF model for a single day from CASES-99. Bound.-Layer Meteor., 139, 261281, https://doi.org/10.1007/s10546-010-9583-z.

and -2013 : Analysis of resolved and parameterized vertical transports in convective boundary layers at gray-zone resolutions. J. Atmos. Sci., 70, 3248-3261, https://doi.org/ 10.1175/JAS-D-12-0290.1.

$\longrightarrow$, and - 2015: Representation of the subgrid-scale turbulent transport in convective boundary layers at gray-zone resolutions. Mon. Wea. Rev., 143, 250-271, https://doi.org/10.1175/ MWR-D-14-00116.1.

, and J. Dudhia, 2016: Evaluation of PBL parameterizations in WRF at subkilometer grid spacings: Turbulence statistics in the dry convective boundary layer. Mon. Wea. Rev., 144, 11611177, https://doi.org/10.1175/MWR-D-15-0208.1.

Siebesma, A. P., and J. W. M. Cuijpers, 1995: Evaluation of parametric assumptions for shallow cumulus convection. J. Atmos. Sci., 52, 650-666, https://doi.org/10.1175/1520-0469(1995) $052<0650$ :EOPAFS $>2.0$. CO 2 .

— , P. M. M. Soares, and J. Teixeira, 2007: A combined eddydiffusivity mass-flux approach for the convective boundary layer. J. Atmos. Sci., 64, 1230-1248, https://doi.org/10.1175/JAS3888.1.

Skamarock, W. C., and Coauthors, 2008: A description of the Advanced Research WRF version 3. NCAR Tech. Note NCAR/TN-475+STR, 113 pp., https://doi.org/10.5065/ D68S4MVH.

Smagorinsky, J., 1963: General circulation experiments with the primitive equations: I. The basic experiment. Mon. Wea. Rev., 91, 99-164, https://doi.org/10.1175/1520-0493(1963)091<0099: GCEWTP $>2.3 . \mathrm{CO} ; 2$.

Thompson, G., P. R. Field, R. M. Rasmussen, and W. D. Hall, 2008: Explicit forecasts of winter precipitation using an improved bulk microphysics scheme. Part II: Implementation of a new snow parameterization. Mon. Wea. Rev., 136, 5095-5115, https://doi.org/10.1175/2008MWR2387.1.

Trier, S. B., F. Chen, and K. W. Manning, 2004: A study of convection initiation in a mesoscale model using high-resolution land surface initial conditions. Mon. Wea. Rev., 132, 29542976, https://doi.org/10.1175/MWR2839.1.

Troen, I., and L. Mahrt, 1986: A simple model of the atmospheric boundary layer: Sensitivity to surface evaporation. Bound.-Layer Meteor., 37, 129-148, https://doi.org/10.1007/ BF00122760.

Wyngaard, J. C., 2004: Toward numerical modeling in the "terra incognita." J. Atmos. Sci., 61, 1816-1826, https://doi.org/10.1175/ 1520-0469(2004)061<1816:TNMITT>2.0.CO;2.

$\mathrm{Xu}, \mathrm{Q} ., 1988$ : A formula for eddy viscosity in the presence of moist symmetric instability. J. Atmos. Sci., 45, 5-8, https://doi.org/ 10.1175/1520-0469(1988)045<0005:AFEVIT>2.0.CO;2.

Xue, M., 2000: High-order monotonic numerical diffusion and smoothing. Mon. Wea. Rev., 128, 2853-2864, https://doi.org/ 10.1175/1520-0493(2000)128<2853:HOMNDA > 2.0.CO;2. 